\title{
The equivalence of several conjectures on independence of $\ell$
}

\author{
Remy van Dobben de Bruyn
}

\begin{abstract}
We consider several conjectures on the independence of $\ell$ of the étale cohomology of (singular, open) varieties over $\overline{\mathbf{F}}_{p}$. The main result is that independence of $\ell$ of the Betti numbers $h_{\mathrm{c}}^{i}\left(X, \mathbf{Q}_{\ell}\right)$ for arbitrary varieties is equivalent to independence of $\ell$ of homological equivalence $\sim_{\text {hom, } \ell}$ for cycles on smooth projective varieties. We give several other equivalent statements. As a surprising consequence, we prove that independence of $\ell$ of Betti numbers for smooth quasi-projective varieties implies the same result for arbitrary separated finite type $k$-schemes.
\end{abstract}

Keywords. Mathematics; algebraic geometry; arithmetic geometry; étale cohomology; positive characteristic; motives; independence of $\ell$

2020 Mathematics Subject Classification. 14F20 (primary); 14F30, 14C15, 14 G15 (secondary)

\section{[Français]}

\section{L'équivalence de plusieurs conjectures sur l'indépendance de $\ell$}

Résumé. Nous considérons plusieurs conjectures sur l'indépendance de $\ell$ pour la cohomologie étale des variétés (singulières, ouvertes) sur $\overline{\mathbf{F}}_{p}$. Le résultat principal est que l'indépendance de $\ell$ des nombres de Betti $h_{\mathrm{c}}^{i}\left(X, \mathbf{Q}_{\ell}\right)$ pour les variétés arbitraires est équivalente à l'indépendance

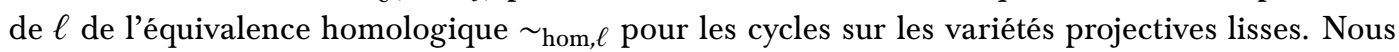
donnons plusieurs autres énoncés équivalents. Comme conséquence surprenante, nous prouvons que l'indépendance de $\ell$ des nombres de Betti pour les variétés quasi-projectives lisses implique le même résultat pour les $k$-schémas séparés de type fini.

Received by the Editors on June 12, 2019, and in final form on August 1, 2020.

Accepted on September 2, 2020.

Remy van Dobben de Bruyn

Department of Mathematics, Princeton University, Princeton, NJ 08544, United States of America

Institute for Advanced Study, Princeton, NJ 08540, United States of America

e-mail:rdobben@math.princeton.edu

Part of this work was carried out with support of the Oswald Veblen Fund at the Institute for Advanced Study.

(C) by the author(s)

This work is licensed under http://creativecommons.org/licenses/by-sa/4.0/ 


\section{Contents}

Introduction . . . . . . . . . . . . . . . . . . . . . . . . . . . . . . . . . 2

1. Pure motives and Weil cohomology theories . . . . . . . . . . . . . . . . . . . 5

2. Simplicial topoi and mapping cones . . . . . . . . . . . . . . . . . . . . . . . . . . 8

3. All-or-nothing motives and additional axioms . . . . . . . . . . . . . . . . . . 11

4. Independence of Weil cohomology theory . . . . . . . . . . . . . . . . . . . . 15

5. Cycle classes and ranks

6. Ranks and dimensions. . . . . . . . . . . . . . . . . . . . . . . . . . . . 18

7. Dimensions and cycle class maps . . . . . . . . . . . . . . . . . . . . . . . 22

References. . . . . . . . . . . . . . . . . . . . . . . 25

\section{Introduction}

Let $k$ be a field, and let $X$ be a $k$-variety. For every prime number $\ell$ invertible in $k$, there is an associated étale cohomology group $H_{\mathrm{c}}^{*}\left(X_{\bar{k}}, \mathbf{Q}_{\ell}\right)$ defined using the geometry of $\ell$-power degree covers of $X$. The main question we want to consider is the following.

Question 1. Given a variety $X$ over a field $k$, is the dimension $h_{\mathrm{c}}^{i}\left(X_{\bar{k}}, \mathbf{Q}_{\ell}\right)$ of $H_{\mathrm{c}}^{i}\left(X_{\bar{k}}, \mathbf{Q}_{\ell}\right)$ independent of the prime $\ell$ ?

If $k=\mathrm{C}$ and $X$ is smooth, this easily follows from the functorial comparison isomorphisms [SGA4 $4_{\text {III }}$, exp. XI, th. 4.4(iii)]

$$
H_{\text {êt }}^{*}\left(X, \mathbf{Q}_{\ell}\right) \cong H_{\text {sing }}^{*}(X(\mathbf{C}), \mathbf{Q}) \otimes_{\mathbf{Q}} \mathbf{Q}_{\ell} .
$$

The result for arbitrary $X$ over $C$ can be deduced from this using hypercoverings, cf. [Del74b, 6.2.8]. The Lefschetz principle proves the result for any field $k$ of characteristic 0 , since étale cohomology is insensitive to extensions of algebraically closed fields [SGA4

On the other hand, if $k$ is finite and $X$ is smooth and proper, then the Weil conjectures [Del74a], [Del80] imply that $h_{\mathrm{et}}^{i}\left(X_{\bar{k}}, \mathbf{Q}_{\ell}\right)$ can be read off from the zeta function of $X$, and thus does not depend on $\ell$. The question for arbitrary $k$-varieties $X$ is a well-known open problem [Kat94, p. 28, (2a)], [Ill06, 3.5(c)].

The homological standard conjecture [Gro69, §4, Remarks (3)] is known to imply the result in the following two cases:

(i) $X$ is proper;

(ii) $X$ is the complement of a simple normal crossings divisor $D$ in a smooth projective variety $\bar{X}$.

Indeed, (ii) is explained in [Kat94, p. 28-29], and (i) is an application of de Jong's alterations [dJo96]. Even assuming the homological standard conjecture, the result for an arbitrary variety $X$ does not seem to appear in the literature (although it may have been known to experts). One cannot simply combine the arguments of (i) and (ii); see Remark 6.7.

We improve these conditional results in three ways:

- we replace the homological standard conjecture by a weaker assumption; 
- we prove independence of $\ell$ of $h_{\mathrm{c}}^{i}\left(X_{\bar{k}}, \mathbf{Q}_{\ell}\right)$ for every separated finite type $k$-scheme $X$;

- we prove a converse as well.

Theorem 1. Let $k$ be an algebraically closed field. If $k=\overline{\mathbf{F}}_{p}$, then the following are equivalent:

(1) For every smooth projective $k$-scheme $X$, the kernel of the cycle class map cl: $\mathrm{CH}_{\mathbf{Q}}^{*}(X) \rightarrow H^{*}\left(X, \mathbf{Q}_{\ell}\right)$ is independent of $\ell$;

(2) For all smooth projective $k$-schemes $X$ and $Y$, any $\alpha \in \mathrm{CH}_{\mathbf{Q}}^{*}(X \times Y)$, and any $i$, the rank of $\alpha_{*}: H^{i}\left(X, \mathbf{Q}_{\ell}\right) \rightarrow H^{*}\left(Y, \mathbf{Q}_{\ell}\right)^{1}$ is independent of $\ell ;$

(3a) For every separated finite type $k$-scheme $X$ and any $i$, the dimension of $H_{\mathrm{c}}^{i}\left(X, \mathrm{Q}_{\ell}\right)$ is independent of $\ell$;

(3b) For every smooth quasi-projective $k$-scheme $X$ and any $i$, the dimension of $H_{\mathrm{c}}^{i}\left(X, \mathbf{Q}_{\ell}\right)$ is independent of $\ell$. Moreover, if these hold when $k=\overline{\mathbf{F}}_{p}$ for some prime $p$ (resp. for every prime $p$ ), then they hold over any algebraically closed field of characteristic $p$ (resp. any algebraically closed field).

This result is given in Theorem 4.6 and Remark 4.8 below. This gives many new angles to the independence of $\ell$ question. The implication $(3 b) \Rightarrow(3 a)$ is particularly surprising; the proof goes through (1) and (2).

We also have an extension to crystalline cohomology. In fact, we work with an arbitrary Weil cohomology theory (see Definition 1.1) satisfying some additional axioms (see Axiom 3.6), at the expense of restricting to $k=\overline{\mathbf{F}}_{p}$. (Developing 'Weil cohomology theories with specialisation' would take us too far afield.)

However, our methods do not say anything about independence of $\ell$ of the dimensions $h^{i}\left(X, \mathbf{Q}_{\ell}\right)$ of the (usual) cohomology groups $H^{i}\left(X, \mathbf{Q}_{\ell}\right)$, except in the proper (resp. smooth) case where it coincides with (resp. is dual to) compactly supported cohomology.

The idea of $(1) \Rightarrow(2)$ is that the rank of a linear map $f: V \rightarrow W$ is the largest $r \in \mathbf{Z}_{\geq 0}$ such that $\bigwedge^{r} f \neq 0$. Although the functors $H^{*}\left(-, \mathbf{Q}_{\ell}\right): \mathbf{M o t}_{k} \rightarrow \mathbf{g V e c}$ do not preserve wedge products (see Remark $1.10)$, algebraicity of the Künneth projectors [KM74] decomposes a cycle $\alpha \in \mathrm{CH}_{\mathbf{Q}}^{*}(X \times Y)$ as $\alpha_{\text {even }} \oplus \alpha_{\text {odd }}$. Then $\bigwedge^{r} \alpha_{\text {even }}$ (resp. $S^{r} \alpha_{\text {odd }}$ ) acts on cohomology as $\bigwedge^{r} \alpha_{\text {even,* }}$ (resp. $\bigwedge^{r} \alpha_{\text {odd, } *}$ ), so the rank of the map $\alpha_{*}: H^{*}\left(X, \mathbf{Q}_{\ell}\right) \rightarrow H^{*}\left(Y, \mathbf{Q}_{\ell}\right)$ is determined by the vanishing or nonvanishing of $\operatorname{cl}\left(\wedge^{r} \alpha_{\text {even }}\right)$ and $\operatorname{cl}\left(S^{r} \alpha_{\text {odd }}\right)$ for various $r$.

To prove $(2) \Rightarrow(3 a)$, we use a variant of the classical hypercovering argument [SGA4 $4_{\text {II }}$, exp. $\mathrm{V}^{\text {bis }}$ ], [Del74b, $\S 5,6]:$ if $X \bullet \rightarrow X$ is a proper hypercovering, then there is a hypercohomology spectral sequence

$$
E_{1}^{p, q}=H^{q}\left(X_{p}, \mathbf{Q}_{\ell}\right) \Rightarrow H^{p+q}\left(X, \mathbf{Q}_{\ell}\right) .
$$

If each $X_{p}$ is smooth projective, then (1) degenerates on the $E_{2}$ page for weight reasons, so $h^{i}\left(X, \mathbf{Q}_{\ell}\right)$ is determined by the ranks of the maps on the $E_{1}$ page.

However, a proper hypercovering by smooth projective schemes can only exist if $X$ is proper. In general, again using de Jong's alterations [dJo96], one can construct a proper hypercovering $X_{\bullet} \rightarrow X$ where each $X_{i}$ is the complement of a simple normal crossings divisor $Z_{i}$ in a smooth projective $k$-scheme $\bar{X}_{i}$. There is a different spectral sequence [Kat94, p. 28-29] computing the compactly supported cohomology of $X_{i}$ in terms of $\bar{X}_{i}$ and the components of $Z_{i}$; its dual then computes the cohomology of $X_{i}$. However, if we then compute (1), the purity argument no longer applies.

Instead, we choose a compactification $X \rightarrow \bar{X}$ first, with closed complement $V$, and we produce a morphism of simplicial schemes $v_{\bullet}: V_{\bullet} \rightarrow \bar{X}_{\bullet}$, where $V_{\bullet}\left(\right.$ resp. $\left.\bar{X}_{\bullet}\right)$ is a hypercovering of $V($ resp. $\bar{X})$. Then

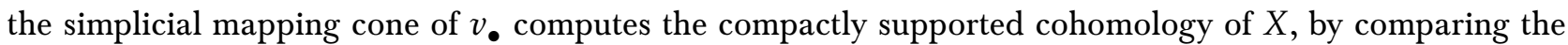
long exact sequence for the mapping cone with that for the triple $(\bar{X}, X, V)$. This allows us to apply the purity theorem as in the argument above for $X$ proper, which finishes the proof of $(2) \Rightarrow(3 a)$.

Finally, for the implication $(3 b) \Rightarrow(1)$ we prove that any cycle $\alpha \in \mathrm{CH}_{d}(X)$ can be written as a difference $\left[Z_{1}\right]-\left[Z_{2}\right]$ with the $Z_{i}$ irreducible; see Corollary 7.5. Letting $U$ be the complement of $Z_{1} \cup Z_{2}$, we relate

\footnotetext{
${ }^{1}$ We do not write $H^{i}\left(Y, \mathbf{Q}_{\ell}\right)$ because $\alpha_{*}$ does not always take $H^{i}$ to $H^{i}$; see Definition 1.7.
} 
the vanishing of $\operatorname{cl}(\alpha)$ to the dimension of $H_{\mathrm{c}}^{2 d+1}\left(U, \mathbf{Q}_{\ell}\right)$. There are only two possible cases depending on whether $\operatorname{cl}\left(Z_{1}\right)$ and $\mathrm{cl}\left(Z_{2}\right)$ are linearly independent or linearly dependent; in the latter case the linear relation is determined by intersection numbers.

\section{Outline of the paper}

In Section 1 we give a brief review of Weil cohomology theories (Definition 1.1) and pure motives (Definition 1.5). Section 2 contains a review of simplicial schemes and mapping cones, which play a $\operatorname{role}^{2}$ in $(2) \Rightarrow(3 a)$. In Section 3 we state the additional axioms on our Weil cohomology theory for the arguments to work; see Axiom 3.6.

The main theorem will be stated in Section 4 (see Theorem 4.6). We then proceed to prove the implications of Theorem 4.6 as outlined in the introduction above, in the following cyclic order:

$$
(1) \Longrightarrow(2) \Longrightarrow(3 a) \Longrightarrow(3 b) \Longrightarrow(1) \text {. }
$$

The implication $(3 a) \Rightarrow(3 b)$ is trivial; each of the others will take up one section (Section 5 , Section 6 , and Section 7 respectively).

\section{Notation and conventions}

If $k$ is a field, then a $k$-variety will mean a finite type, separated, geometrically integral $k$-scheme. A pair $(X, H)$ is called a projective $k$-scheme if $X$ is a projective $k$-scheme and $H$ a very ample divisor on $X$.

In the main theorems, the base field $k$ will be assumed algebraically closed, because standard references on Weil cohomology theories have this running assumption, and establishing the general framework would take us too far astray.

The category of smooth projective $k$-varieties will be denoted by SmPrVar ${ }_{k}$, and the category of smooth projective $k$-schemes will be denoted by $\operatorname{SmPr}_{k}$. The latter can be obtained from the former as the category of formal finite coproducts (if $k$ is algebraically closed), cf. Example 3.5. The category of Chow motives is denoted by $\operatorname{Mot}_{k}$; its definition will be recalled in Definition 1.5. Morphisms in this category are typically denoted by $\alpha: X \vdash Y$.

If $K$ is a field, then $\operatorname{Vec}_{K}$ denotes the category of $K$-vector spaces, $\mathbf{g V e c}_{K}$ the category of $Z$-graded $K$-vector spaces, and $\mathbf{g A l g} \mathbf{g}_{K}$ the category of $\mathbf{Z}$-graded (unital, associative) $K$-algebras. The objects of $\mathbf{g A l g}_{K}$ we encounter will always be graded-commutative and vanish in negative degrees.

We write $\mathbf{A b}(\mathscr{C})$ for the category of abelian group objects in a category $\mathscr{C}$ with finite products. All topoi $^{3}$ will be Grothendieck topoi, i.e. the topos of sheaves (of sets) on a small category with a Grothendieck topology (or pretopology). We write Topos for the (strict) 2-category of topoi, whose objects are topoi, whose 1 -morphisms are (geometric) morphisms of topoi, and whose 2-morphisms are natural transformations between the inverse image functors (equivalently, between the direct image functors).

We write Shv for the (strict) 2-category whose objects are pairs $(X, \mathscr{F})$ of a topos $X$ with an abelian object $\mathscr{F}$ in $X$, whose 1 -morphisms $(X, \mathscr{F}) \rightarrow(Y, \mathscr{G})$ are pairs $(f, \phi)$ of a 1 -morphism $f: X \rightarrow Y$ and a morphism $\phi: f^{*} \mathscr{G} \rightarrow \mathscr{F}$ of abelian objects, and whose 2-morphisms $(f, \phi) \rightarrow(g, \psi)$ are given by natural transformations $\eta: f^{*} \Rightarrow g^{*}$ such that $\psi \circ \eta_{\mathscr{G}}=\mathrm{id} \mathrm{F}_{\mathscr{F}} \circ \phi$. We think of it as a "fibred 2-category" Shv $\rightarrow$ Topos, whose fibre above the topos $X$ is $\mathbf{A b}(X)^{\mathrm{op}}$ (with only identity 2-morphisms). In a similar way, we define a category Comp of pairs $(X, K)$ of a topos $X$ with a complex $K$ of abelian objects on $X$.

\footnotetext{
${ }^{2}$ Weizhe Zheng provided an easier argument without mapping cones; see Remark 6.8. We kept the original argument as it proves more, and the techniques might be useful elsewhere.

${ }^{3}$ We work with topoi instead of sites because they have better formal properties, but all arguments could also be carried out using Grothendieck (pre)topologies. We ignore set-theoretic issues; they can for instance be dealt with using universes.
} 


\section{Acknowledgements}

This paper grew out of a conversation with Ashwin Deopurkar on the existence of exterior powers in the category of pure motives. I also thank Raymond Cheng, Johan Commelin, and Johan de Jong for helpful conversations. I am particularly grateful to Weizhe Zheng for providing an alternative and easier proof of $(2) \Rightarrow(3 a)$; see Remark 6.8 . Finally, I thank the referee for many helpful suggestions and corrections.

\section{Pure motives and Weil cohomology theories}

This is a review of the theory of pure motives, cf. e.g. Kleiman [Kle72], Jannsen [Jan92], or Scholl's excellent survey [Sch94]. We also give a brief review of Weil cohomology theories; see [Kle94, §3] for more details. Following standard references, we will assume that $k$ is algebraically closed. Our setup is slightly more general than [Kle94, §3], in that we allow smooth projective $k$-schemes with multiple components.

Definition 1.1. Let $k$ be an algebraically closed field, and let $K$ be a field of characteristic 0. A Weil cohomology theory is a functor $H: \mathbf{S m P r}_{k}^{\mathrm{op}} \rightarrow \mathbf{g A l g}_{K}$ satisfying the following axioms.

(W1) Each $H^{i}(X)$ is finite-dimensional and vanishes for $i<0$ and $i>2 \operatorname{dim} X$;

(W2) There is a trace map $\operatorname{tr}_{X}: H^{2 \operatorname{dim} X}(X) \rightarrow K$ that is an isomorphism if $X$ is irreducible, and takes 1 to 1 if $X=$ Spec $k$. If all components of $X$ have the same dimension $d$, then the natural pairing $H^{i}(X) \times H^{2 d-i}(X) \rightarrow K$ is perfect;

(W3) The projections induce an isomorphism $H^{*}(X) \otimes_{K} H^{*}(Y) \stackrel{\sim}{\rightarrow} H^{*}(X \times Y)$;

(W4) There are cycle class maps $\mathrm{cl}: \mathrm{CH}_{\mathbf{Q}}^{i}(X) \rightarrow H^{2 i}(X)$. It is a ring homomorphism functorial for pullback and pushforward, where pushforward for $H$ is defined using (W2);

(W5) The weak Lefschetz theorem holds;

(W6) The hard Lefschetz theorem holds;

(W7) $H$ preserves products, i.e. $H(X) \cong \prod_{i} H\left(X_{i}\right)$ if $X=\bigsqcup_{i} X_{i}$. Moreover, this isomorphism identifies $\operatorname{tr}_{X}$ with $\sum_{i} \operatorname{tr}_{X_{i}}$.

Example 1.2. For every prime $\ell$ invertible in $k$, the $\ell$-adic étale cohomology gives a Weil cohomology theory [SGA4 ${ }_{\text {III }}$, Del74a, Del80].

Example 1.3. For a perfect field $k$ of positive characteristic $p$ with Witt ring $W(k)$ with field of fractions $K$, crystalline cohomology $H_{\text {cris }}^{i}(X / K)$ is a Weil cohomology theory [Ber74], [KM74, Corollary 1(2)], [Gro85], [GM87]. See also [Ill94] for an expository account and additional references.

Let $\sim$ be an adequate equivalence relation (cf. [Sam60]) finer than homological equivalence for every Weil cohomology theory, e.g. $\sim$ is rational, algebraic, or smash-nilpotent equivalence. For a variety $X$ and $i \in \mathbf{Z}_{\geq 0}$, we will write $\mathrm{CH}^{i}(X)$ for algebraic cycles of codimension $i$ modulo $\sim$, and $\mathrm{CH}_{\mathbf{Q}}^{i}(X)$ for $\mathrm{CH}^{i}(X) \otimes \mathbf{Q}$.

We will omit further mention of $\sim$ unless it plays a role in the arguments.

Definition 1.4. Let $X$ and $Y$ be smooth projective $k$-schemes, and assume that $X$ has connected components $X_{1}, \ldots, X_{m}$ of dimensions $d_{1}, \ldots, d_{m}$ respectively. Then the group of correspondences of degree $r$ (modulo $\sim$ ) from $X$ to $Y$ is

$$
\operatorname{Corr}^{r}(X, Y):=\bigoplus_{i=1}^{m} \mathrm{CH}_{\mathrm{Q}}^{d_{i}+r}\left(X_{i} \times Y\right)
$$


An element $\alpha \in \operatorname{Corr}^{r}(X, Y)$ is a correspondence from $X$ to $Y$, and is denoted $\alpha: X \vdash Y$. There is a natural composition of correspondences:

$$
\begin{aligned}
\operatorname{Corr}^{r}(X, Y) \times \operatorname{Corr}^{s}(Y, Z) & \rightarrow \operatorname{Corr}^{r+s}(X, Z) \\
(\alpha, \beta) & \mapsto \beta \circ \alpha .
\end{aligned}
$$

If the degree $r$ in the superscript is omitted, it will be assumed 0 .

Definition 1.5. The category of Chow motives (modulo $\sim$ ) is the category whose objects are triples $(X, p, m)$, where $X$ is a smooth projective $k$-scheme, $p \in \operatorname{Corr}(X, X)$ a projector (i.e. $p^{2}=p$ ), and $m \in \mathbf{Z}$ an integer. Morphisms $(X, p, m) \vdash(Y, q, n)$ from $(X, p, m)$ to $(Y, q, n)$ are given by

$$
\begin{aligned}
\operatorname{Hom}((X, p, m),(Y, q, n)) & =q \operatorname{Corr}^{n-m}(X, Y) p \\
& =\left\{\alpha \in \operatorname{Corr}^{n-m}(X, Y) \mid \alpha p=\alpha=q \alpha\right\} .
\end{aligned}
$$

We denote the category of Chow motives by $\operatorname{Mot}_{k}$. The motive (Speck,id,-1) is called the Lefschetz motive, and is denoted by $\mathbf{L}$. We write $\mathbf{L}^{n}$ for (Spec $\left.k, \mathrm{id},-n\right)$, which can also be defined as $\mathbf{L}^{\otimes n}$ using the tensor product of Remark 1.9.

Remark 1.6. There is a functor $\operatorname{SmPr}_{k}^{\mathrm{op}} \rightarrow \operatorname{Mot}_{k}$ associating to every smooth projective $k$-scheme $X$ the motive $(X$, id, 0$)$, and to every map $f: X \rightarrow Y$ the (class of the) graph $\Gamma_{f} \in \operatorname{Corr}(Y, X)$.

Definition 1.7. If $H$ is a Weil cohomology theory with coefficient field $K$, then Poincaré duality gives an isomorphism

$$
\begin{aligned}
H^{*}(X) & \longrightarrow H^{*}(X)^{\vee} \\
v & \longmapsto\left(w \mapsto \int v \smile w\right)
\end{aligned}
$$

Together with the Künneth formula this gives isomorphisms

$$
H^{*}(X \times Y) \cong H^{*}(X) \otimes H^{*}(Y) \cong H^{*}(X)^{\vee} \otimes H^{*}(Y)=\operatorname{Hom}\left(H^{*}(X), H^{*}(Y)\right)
$$

If $\alpha \in \operatorname{Corr}^{r}(X, Y)$, then under these isomorphisms $\operatorname{cl}(\alpha)$ induces a pushforward

$$
\alpha_{*}: H^{i}(X) \rightarrow H^{i+2 r}(Y)
$$

In particular, a projector $p \in \operatorname{Corr}(X, X)$ induces a projector on $H^{*}$, and we extend $H$ to a functor $\mathbf{M o t}_{k} \rightarrow \mathbf{g V e c}_{K}$ by setting

$$
H^{*}(X, p, m):=p H^{*}(X)[2 m]
$$

where for a graded vector space $V=\bigoplus_{i} V^{i}$, we set $V^{i}[m]=V^{i+m}$. 
Given a morphism $\alpha:(X, p, m) \vdash(Y, q, n)$, we define $H \alpha$ to be the graded map given by the pushforward $\alpha_{*}: p H^{i+2 m}(X) \rightarrow q H^{i+2 n}(Y)$ as in (1.2).

Some Weil cohomology theories have further structure (e.g. a Hodge structure or a Galois action), and these structures are typically preserved by pushforward along cycles (if everything is given the correct 'Tate twist'). We will consider this additional structure understood, and we will not use separate notation for the corresponding enriched functor.

Example 1.8. The cohomology $H^{i}(\mathbf{L})$ of the Lefschetz motive is 0 if $i \neq 2$ and one-dimensional if $i=2$. It's often thought of as the compactly supported cohomology of $\mathbf{A}^{1}$ (or the reduced cohomology of $\mathbf{P}^{1}$ ), and is equipped with the corresponding Galois action or Hodge structure.

Remark 1.9. The category $\operatorname{Mot}_{k}$ has a tensor product given by

$$
(X, p, m) \otimes(Y, q, n)=(X \times Y, p \otimes q, m+n) .
$$

Thus, we also get symmetric and alternating products $S^{n}$ and $\bigwedge^{n}$ by considering the projectors $\frac{1}{n !} \sum_{\sigma} \sigma$ and $\frac{1}{n !} \sum_{\sigma}(-1)^{\sigma} \sigma$ respectively on $X^{n}$.

Remark 1.10. If $H$ is a Weil cohomology theory with coefficient field $K$, then the functor $H: \mathbf{M o t}_{k} \rightarrow \mathbf{g V e c}_{K}$ is a tensor functor if we equip $\mathbf{g V e c}_{K}$ with the tensor product as in super vector spaces: on objects, it is given by the usual graded tensor product, but the swap is given by

$$
\begin{aligned}
\tau_{V, W}: V \otimes W & \rightarrow W \otimes V \\
v_{i} \otimes w_{j} & \mapsto(-1)^{i j} w_{j} \otimes v_{i},
\end{aligned}
$$

for homogeneous elements $v_{i} \in V^{i}, w_{j} \in W^{j}$. To see that this makes $H$ into a tensor functor, note that the Künneth isomorphism is given by the map

$$
\begin{aligned}
H^{*}(X) \otimes H^{*}(Y) & \rightarrow H^{*}(X \times Y) \\
\alpha \otimes \beta & \mapsto \pi_{X}^{*} \alpha \smile \pi_{Y}^{*} \beta,
\end{aligned}
$$

which under swapping $X$ and $Y$ picks up a factor $(-1)^{\operatorname{deg} \alpha \operatorname{deg} \beta}$.

Remark 1.11. In particular, if $H^{*}(X)=H^{\text {even }} \oplus H^{\text {odd }}$, then

$$
H^{*}\left(S^{r} X\right)=S^{r}\left(H^{*}(X)\right)=\bigoplus_{i+j=r} S^{i} H^{\text {even }} \otimes \bigwedge^{j} H^{\text {odd }},
$$

and conversely

$$
H^{*}\left(\bigwedge^{r} X\right)=\bigwedge^{r}\left(H^{*}(X)\right)=\bigoplus_{i+j=r} \bigwedge^{i} H^{\text {even }} \otimes S^{j} H^{\text {odd }}
$$

Indeed, because of the sign in $\tau_{V, W}$, the symmetriser and antisymmetriser get swapped in odd degree.

Remark 1.12. The category $\operatorname{Mot}_{k}$ has binary biproducts; for example

$$
(X, p, n) \oplus(Y, q, n)=(X \amalg Y, p \oplus q, n) .
$$

One can construct $(X, p, m) \oplus(Y, q, n)$ when $m \neq n$ as well, by replacing $X$ by $X \times \mathbf{P}^{n-m}$ or $Y$ by $Y \times \mathbf{P}^{m-n}$, using that $\mathbf{L}^{d}=(\mathrm{pt}, \mathrm{id},-d)$ is a summand of $\left(\mathbf{P}^{d}, \mathrm{id}, 0\right)$ for $d \geq 0$ (see e.g. [Sch94, 1.13]), and using the tensor product of Remark 1.9 to reduce to the case $m=n$.

However, the category $\mathbf{M o t}_{k}$ is not in general abelian, and in fact for our choices of $\sim$ this is either false or open; see for example [Sch94, Corollary 3.5] for the case where $\sim$ is rational equivalence and $k$ is not isomorphic to $\overline{\mathbf{F}}_{p}$.

Lemma 1.13. Let $X$ and $Y$ be smooth projective schemes over a field $k$, and let $\alpha \in \operatorname{Corr}(X, Y)$. Then the map $\alpha_{*}: H^{*}(X) \rightarrow H^{*}(Y)$ is 0 if and only if $\operatorname{cl}(\alpha)=0 \in H^{*}(X \times Y)$. 
Proof. This is clear from the definition of $\alpha_{*}$, cf. Definition 1.7.

Lemma 1.14. Let $X$ be an $n$-dimensional smooth projective $k$-scheme, and let $\alpha \in \operatorname{Corr}(X, X)$. Then

$$
\sum_{i=0}^{2 n}(-1)^{i} \operatorname{tr}\left(\left.\alpha_{*}\right|_{H^{i}(X)}\right)=\alpha \cdot\left[\Delta_{X}\right],
$$

where $\Delta_{X} \subseteq X \times X$ is the diagonal. In particular, it is a rational number that does not depend on $H$.

Proof. The first statement is well-known, and the second follows.

Corollary 1.15. Let $X$ be a smooth projective $k$-variety such that $\operatorname{Kün}(X)$ holds (see Definition 4.1). Let $\alpha \in \operatorname{Corr}(X, X)$. Then the characteristic polynomial of $\alpha$ on $H^{i}(X)$ is in $\mathrm{Q}[t]$, and is independent of the Weil cohomology theory $H$.

Proof. One easily checks that the coefficients of the characteristic polynomial

$$
P_{A}(t)=\operatorname{det}(t \cdot I-A)=t^{n}+c_{n-1} t^{n-1}+\ldots+c_{1} t+c_{0}
$$

of an endomorphism $A$ on an $n$-dimensional vector space $V$ are given by

$$
c_{j}=(-1)^{n-j} \operatorname{tr}\left(\wedge^{n-j} A\right) .
$$

Hence, if $p_{i} \in \operatorname{Corr}(X, X)$ denotes the $i$-th Künneth projector, then applying Lemma 1.14 to $\bigwedge^{n-j}\left(p_{i} \circ \alpha\right)$ gives the result.

\section{Simplicial topoi and mapping cones}

We will use the following (possibly non-standard) terminology:

Definition 2.1. Let $D$ be a small category, and let $\mathscr{C}$ be a category. Then a $D$-object in $\mathscr{C}$ is a functor $D^{\text {op }} \rightarrow \mathscr{C}$. If $D$ is the category $\Delta_{+}$of finite (totally) ordered sets with monotone maps (resp. the subcategory $\Delta$ of nonempty objects), then a $D$-object is an augmented simplicial object (resp. simplicial object) of $\mathscr{C}$.

If $X_{\bullet}$ is an (augmented) simplicial object, then $X_{n}$ denotes the value of $X_{\bullet}$ on the set $[n]=\{0, \ldots, n\}$. Giving an augmented simplicial object $\left(X_{n}\right)_{n \geq-1}$ is equivalent to giving a simplicial object $X_{\bullet}=\left(X_{n}\right)_{n \geq 0}$ together with a map $X_{\bullet} \rightarrow X_{-1}$ to the constant simplicial object with value $X_{-1}$.

Remark 2.2. If $\mathscr{C}$ is a 2-category (e.g. Topos or Shv; see Notation), then the correct definition of a $D$-object is a pseudofunctor $X_{\bullet}: D^{\text {op }} \rightarrow \mathscr{C}$. By the 'Grothendieck construction', this should correspond to some sort of fibred object. For example, a $D$-topos corresponds to a functor $F: X \rightarrow D$ that is a fibration and cofibration, such that each fibre $X_{i}$ is a topos, and for any morphism $\phi: i \rightarrow j$ in $D$ the pair $\left(f_{*}, f^{*}\right)=\left(\phi^{*}, \phi_{*}\right)$ is a morphism of topoi $f: X_{j} \rightarrow X_{i}$. This is the definition of $D$-topos in [SGA4 $4_{\mathrm{II}}$, exp. $\mathrm{V}^{\text {bis }}$, déf. 1.2.1].

By [SGA4 ${ }_{\text {II }}$, exp. $V^{\text {bis }}$, déf. 1.2.8 and prop. 1.2.12], this gives rise to a total topos $\Gamma(X)$. Abelian objects in $\Gamma(X)$ correspond to enrichments of the pseudofunctor $X_{\bullet}: D^{\text {op }} \rightarrow$ Topos through $D^{\text {op }} \rightarrow$ Shv, and similarly for complexes.

Remark 2.3. Let $f: D^{\prime} \rightarrow D$ be a functor, and let $X \rightarrow D$ a $D$-topos. Then the pullback $D^{\prime} \times_{D} X \rightarrow D^{\prime}$ is a $D^{\prime}$-topos, and $f^{*}: \Gamma(X) \rightarrow \Gamma\left(D^{\prime} \times_{D} X\right)$ has left and right adjoints $f_{!}$and $f^{*}$ [SGA4 $4_{\text {II }}$, exp. V $^{\text {bis }}$, prop. 1.2.9]; in particular, $\left(f_{*}, f^{*}\right): \Gamma\left(D^{\prime} \times_{D} X\right) \rightarrow \Gamma(X)$ is a morphism of topoi.

For an inclusion $e_{d}: d \rightarrow D$ of an object $d \in D$, we write $\left.(-)\right|_{X_{d}}$ instead of $e_{d}^{*}$. In this case [SGA4 II exp. $\mathrm{V}^{\text {bis }}$, cor. 1.2.11 and prop. 1.3.7] show that $e_{d, !}$ is given on abelian objects by

$$
e_{d, !}(\mathscr{F})\left(d^{\prime}\right)=\bigoplus_{\alpha: d \rightarrow d^{\prime}} \alpha_{*}(\mathscr{F})
$$

Note that $e_{d, !}$ is exact, since $\alpha_{*}$ is exact (since $\left(f_{*}, f^{*}\right)=\left(\alpha^{*}, \alpha_{*}\right)$ is a morphism of topoi), direct sums are exact [SGA4 ${ }_{\mathrm{I}}$, exp. II, prop. 6.7], and exactness in $\mathbf{A b}(\boldsymbol{\Gamma}(X))$ is determined pointwise. 
Remark 2.4. If $D=\Delta$, then following the notation of simplicial topological spaces we will write $\mathbf{A b}\left(X_{\bullet}\right)$ (resp. $\left.\mathbf{C h}\left(X_{\bullet}\right)\right)$ for $\mathbf{A b}(\boldsymbol{\Gamma}(X))$ (resp. $\left.\mathbf{C h}(\boldsymbol{\Gamma}(X))\right)$ and write $H^{i}\left(X_{\bullet}, K\right)$ for $H^{i}(\boldsymbol{\Gamma}(X), K)$. For a bounded below complex ${ }^{4}$ on $X_{\bullet}$, there is a spectral sequence

$$
E_{1}^{p, q}=H^{q}\left(X_{p},\left.K\right|_{X_{p}}\right) \Rightarrow H^{p+q}\left(X_{\bullet}, K\right),
$$

whose $E_{1}$ page is the alternating face complex on the cosimplicial abelian groups $H^{q}\left(X_{*},\left.K\right|_{X_{*}}\right)$; see for example [SGA4 $4_{\mathrm{II}}$, exp. $\mathrm{V}^{\text {bis }}$, cor. 2.3.7 and 2.3.9]. For computation, it's useful to have a refined version of this statement:

Lemma 2.5. Let $X_{\bullet}$ be a simplicial topos, and let $K$ be a bounded below complex on $X_{\bullet}$ with $H^{i}\left(X_{n},\left.K^{j}\right|_{X_{n}}\right)=0$ for all $j \in \mathbf{Z}, n \in \mathbf{N}$, and $i>0$. Then the complex

$$
\operatorname{Tot}\left(\Gamma\left(X_{*},\left.K^{*}\right|_{*}\right)\right)
$$

computes $R \Gamma\left(X_{\bullet}, K\right)$.

Proof. Let Set $\times \Delta$ be the constant simplicial topos, so $\Gamma(\operatorname{Set} \times \Delta)=[\Delta$, Set $]$. Since Set is terminal in Topos [SGA4 I, exp. IV, §4.3], the constant simplicial topos is the terminal simplicial topos, so we get a functor $\theta: X_{\bullet} \rightarrow$ Set $\times \Delta$.

Again since Set is terminal, the terminal morphism $\Gamma: \Gamma(X) \rightarrow$ Set factors as

$$
\Gamma(X) \stackrel{\Gamma(\theta)}{\longrightarrow}[\Delta, \text { Set }] \stackrel{\varepsilon}{\longrightarrow} \text { Set } .
$$

On the level of derived categories of abelian objects, we get

$$
D^{+}\left(X_{\bullet}\right) \stackrel{R \Gamma(\theta)_{*}}{\longrightarrow} D^{+}([\Delta, \mathbf{A b}]) \stackrel{R \varepsilon_{*}}{\longrightarrow} D^{+}(\mathbf{A b}) .
$$

By [SGA4 $4_{\mathrm{II}}$, exp. $\mathrm{V}^{\text {bis }}$, prop. 1.3.7 and cor. 1.3.12] we have

$$
\left(R^{i} \Gamma(\theta)_{*} K^{j}\right)_{n}=R^{i} \theta_{n, *}\left(\left.K^{j}\right|_{X_{n}}\right)=H^{i}\left(X_{n},\left(\left.K^{j}\right|_{X_{n}}\right)\right)=0
$$

for all $n \in \mathbf{N}$, all $j \in \mathbf{Z}$, and all $i>0$. Thus, all $K^{j}$ are acyclic for $R \Gamma(\theta)_{*}$, so

$$
R \Gamma(\theta)_{*} K=\Gamma(\theta)_{*} K .
$$

The result follows since $R \varepsilon_{*}$ is computed by the totalisation of the alternating face complex [SGA4 $4_{\mathrm{II}}$, exp. $\mathrm{V}^{\text {bis }}$, cor. 2.3.6].

Definition 2.6. Let $\mathscr{C}$ be a category with finite coproducts and a terminal object $*$, and let $f_{\bullet}: Y_{\bullet} \rightarrow X_{\bullet}$ be a morphism of simplicial objects in $\mathscr{C}$. Then the mapping cone $C_{\bullet}(f)$ of $f_{\bullet}$ is the simplicial object in $\mathscr{C}$ constructed as the pushout of the diagram

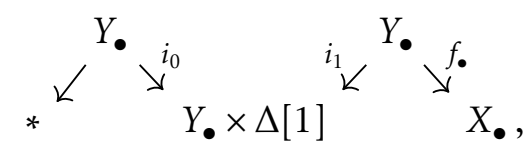

where $Y_{\bullet} \times \Delta[1]$ is the simplicial object of $\mathscr{C}$ defined in [Stacks, Tag $017 \mathrm{C}$ ]. The mapping cone satisfies

$$
C_{n}(f)=* \amalg \underbrace{Y_{n} \amalg \ldots \amalg Y_{n}}_{n} \amalg X_{n} .
$$

\footnotetext{
${ }^{4}$ Note that a complex $K$ on $X_{\bullet}$ is bounded below iff the complexes on the components $X_{i}$ are bounded below uniformly in $i$.
} 
A slightly different construction is given in [Del74b, 6.3.1], but the two are homotopy equivalent as simplicial objects in $\mathscr{C}$. Similarly, the mapping cylinder cyl $(f)$ is the pushout of

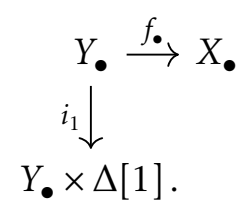

It satisfies

$$
\operatorname{cyl}_{n}(f)=\underbrace{Y_{n} \amalg \ldots \amalg Y_{n}}_{n+1} \amalg X_{n},
$$

and the inclusion $X_{\bullet} \rightarrow \operatorname{cyl}_{\bullet}(f)$ and projection cyl $(f) \stackrel{f_{\bullet}}{\rightarrow} X_{\bullet} \times \Delta[1] \rightarrow X_{\bullet}$ are homotopy inverses as simplicial object in $\mathscr{C}$.

Example 2.7. Let $f_{\bullet}: Y_{\bullet} \rightarrow X_{\bullet}$ be a morphism of simplicial topoi, let $K$ and $L$ be abelian objects (resp. complexes) on $X_{\bullet}$ and $Y_{\bullet}$ respectively, and let $\phi: f_{\bullet}^{*} K \rightarrow L$ be a morphism. Then we may view $\left(f_{\bullet}, \phi\right)$ as a morphism $\left(Y_{\bullet}, L\right) \rightarrow\left(X_{\bullet}, K\right)$ of simplicial objects in Shv (resp. Comp). Thus, there is an abelian object (resp. complex) $C_{\bullet}(\phi)$ on $C_{\bullet}(f)$ such that $\left(C_{\bullet}(f), C_{\bullet}(\phi)\right)$ is the mapping cone in Shv (resp. Comp).

Remark 2.8. The terminal object in Topos is Set [SGA4 $4_{\mathrm{I}}$, exp. IV, §4.3], whose abelian objects are just abelian groups. Moreover, 2-coproducts in Topos are given by the product of categories $\left[\mathrm{SGA} 4_{\mathrm{I}}\right.$, exp. IV, exercice 8.7(bc)]. The terminal object in Shv or Comp is the pair (Set, 0). Thus, in the description of (2.1), the complex $C_{n}(\phi)$ on $C_{n}(f)$ equals 0 on $*=$ Set, equals $\left.L\right|_{Y_{n}}$ on each of the components $Y_{n}$, and equals $\left.K\right|_{X_{n}}$ on the component $X_{n}$.

Lemma 2.9. Let $X_{\bullet}$ and $Y_{\bullet}$ be simplicial topoi, and let $f_{\bullet}: Y_{\bullet} \rightarrow X_{\bullet}$ be a morphism of simplicial topoi. Let $K$ and $L$ be bounded below complexes on $X_{\bullet}$ and $Y_{\bullet}$ respectively, and let $\phi: f_{\bullet}^{*} K \rightarrow L$ be a morphism. Then there is a canonical distinguished triangle

$$
R \Gamma\left(C_{\bullet}(f), C_{\bullet}(\phi)\right) \rightarrow R \Gamma\left(X_{\bullet}, K\right) \rightarrow R \Gamma\left(Y_{\bullet}, L\right) \rightarrow R \Gamma\left(C_{\bullet}(f), C_{\bullet}(\phi)\right)[1]
$$

in $D^{+}(\mathbf{A b})$, functorial in the morphism $(f, \phi):\left(Y_{\bullet}, L\right) \rightarrow\left(X_{\bullet}, K\right)$ of simplicial objects in Comp.

In the setting of simplicial topological spaces, this is [Del74b, 6.3.3].

Proof. View $\left(\phi: f_{\bullet}^{*} K \rightarrow L\right)$ as a bounded below complex $\mathbf{K}$ on the $(\leftarrow)$-topos $\mathbf{X}=(\boldsymbol{\Gamma}(Y) \rightarrow \boldsymbol{\Gamma}(X))$. Let $\mathbf{K} \rightarrow \mathbf{I}$ be an injective resolution. This means that $\mathbf{I}=\left(\psi: f_{\bullet}^{*} I \rightarrow J\right)$ for bounded below complexes of injectives $I$ and $J$ on $X_{\bullet}$ and $Y_{\bullet}$ respectively, together with a commutative square

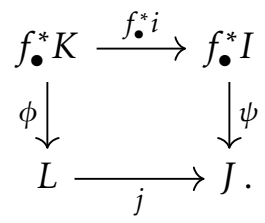

Since $e_{X, !}$ and $e_{Y, !}$ are exact by Remark 2.3, we conclude that $I=e_{X}^{*} \mathbf{I}$ and $J=e_{Y}^{*} \mathbf{I}$ are still injective [Stacks, Tag 015Z]. Since $e_{X}^{*}$ and $e_{Y}^{*}$ are exact, $i: K \rightarrow I$ and $j: L \rightarrow J$ are still resolutions. Similarly, $\left.\left.K\right|_{X_{n}} \rightarrow I\right|_{X_{n}}$ and $\left.\left.L\right|_{Y_{n}} \rightarrow J\right|_{Y_{n}}$ are injective resolutions for any $n \in \mathbf{N}$. Finally, functoriality of the mapping cone and mapping cylinder gives resolutions

$$
\begin{aligned}
C_{\bullet}(\phi) & \rightarrow C_{\bullet}(\psi) \\
\operatorname{cyl}_{\bullet}(\phi) & \rightarrow \operatorname{cyl}_{\bullet}(\psi),
\end{aligned}
$$


where all terms $C_{n}(\psi)$ and $\operatorname{cyl}_{n}(\psi)$ are injective (but we do not know whether $C_{\bullet}(\psi)$ and cyl $(\psi)$ themselves are injective).

From the descriptions of (2.1), (2.2), and Remark 2.8, we see that the maps $Y_{\bullet} \stackrel{i_{0}}{\rightarrow}$ cyl $(f) \rightarrow C_{\bullet}(f)$ induce a termwise split exact sequence

$$
0 \rightarrow \operatorname{Tot}\left(\Gamma\left(C_{*}, C_{\bullet}^{*}(\psi)\right)\right) \rightarrow \operatorname{Tot}\left(\Gamma\left(\operatorname{cyl}_{*}, \operatorname{cyl}_{\bullet}^{*}(\psi)\right)\right) \rightarrow \operatorname{Tot}\left(\Gamma\left(Y_{*}, J^{*}\right)\right) \rightarrow 0 .
$$

The homotopy equivalence $\left(\mathrm{cyl}_{\bullet}, \mathrm{cyl}_{\bullet}(\psi)\right) \simeq\left(X_{\bullet}, I\right)$ induces a chain homotopy equivalence

$$
\operatorname{Tot}\left(\Gamma\left(\operatorname{cyl}_{*}, \operatorname{cyl}_{\bullet}^{*}(\psi)\right)\right) \simeq \operatorname{Tot}\left(\Gamma\left(X_{*}, I^{*}\right)\right)
$$

[Stacks, Tags 019M and 019S]. By Lemma 2.5, the termwise split short exact sequence (2.3) gives the desired distinguished triangle. The obtained sequence does not depend on the choice of $\mathbf{I}$ since any two injective resolutions are (non-canonically) homotopy equivalent [Stacks, Tag 05TG]. For functoriality, let

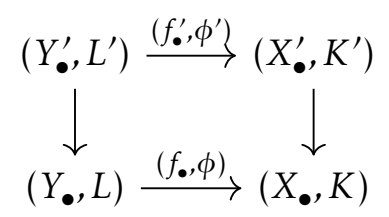

be a commutative diagram of simplicial objects in Comp where $K, K^{\prime}, L$, and $L^{\prime}$ are bounded below. We may view this as a bounded below complex $\mathbf{K}$ on the $\left(\hat{\uparrow}_{\leftarrow}^{+} \uparrow\right)$-topos

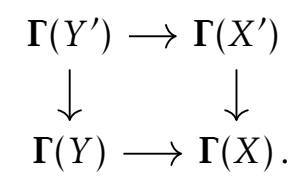

Proceeding as above gives a natural morphism from the sequence (2.3) for $f_{\bullet}$ to the same sequence for $f_{\bullet}^{\prime}$ (which again does not depend on any choices).

\section{All-or-nothing motives and additional axioms}

As in Section 1 we will assume $k$ is an algebraically closed field. We want to apply the previous section in the case where $X_{\bullet}$ is a simplicial scheme, viewed as a simplicial topos with the pro-étale topology [BS15] (resp. the crystalline topology [Ber74]), and $K$ and $L$ are the constant sheaves $\mathbf{Q}_{\ell}$ (resp. the structure sheaf $\left.\mathcal{O}_{X / W(k)}\right)$. The recipe of Definition 2.6 and Remark 2.8 tells us to consider the sheaf on $C_{\bullet}(f)$ given by 0 on the components * of $C_{\bullet}(f)$ and by $\mathbf{Q}_{\ell}$ (resp. $\left.\mathcal{O}_{-/ W(k)}\right)$ on all other components.

Thus, we need to equip the mapping cone of a morphism of simplicial schemes with a mild motivic structure, interpreting the sheaf 0 on $*$ as a variant of the zero motive. This motivates the following ad hoc notion.

Definition 3.1. An all-or-nothing motive $(X, p)$ is a smooth projective $k$-scheme together with a locally constant function $p: X \rightarrow\{0,1\}$. The set of morphisms $f:(X, p) \rightarrow(Y, q)$ of all-or-nothing motives $(X, p)$, $(Y, q)$ whose underlying schemes $X$ and $Y$ are connected is given by

$$
\operatorname{Mor}((X, p),(Y, q))= \begin{cases}\operatorname{Mor}(Y, X), & p=q=1, \\ \{0\}, & p=0, \\ \varnothing, & \text { else, }\end{cases}
$$

with composition given by $f \circ 0=0$. (The contravariance is consistent with Remark 1.6.) 
In general, if $X=\amalg X_{i}$ and $Y=\bigsqcup Y_{j}$ with the $X_{i}$ and $Y_{j}$ irreducible, we set

$$
\operatorname{Mor}((X, p),(Y, q))=\prod_{j} \coprod_{i} \operatorname{Mor}\left(\left(X_{i},\left.p\right|_{X_{i}}\right),\left(Y_{j},\left.q\right|_{Y_{j}}\right)\right) \text {. }
$$

The category of all-or-nothing motives is denoted $\mathbf{M o t}_{k}^{\text {aon }}$.

Remark 3.2. If $X_{i}$ are the components of $X$, then we think of $(X, p)$ as the pure motive $(X, p, 0)$ by identifying $p$ with the projector in $\operatorname{Corr}(X, X)$ given by $0 \in \mathrm{CH}^{*}\left(X_{i} \times X_{i}\right)$ if $\left.p\right|_{X_{i}}=0$ and by $\Delta_{X_{i}} \in \mathrm{CH}^{*}\left(X_{i} \times X_{i}\right)$ if $\left.p\right|_{X_{i}}=1$. This gives a factorisation $\operatorname{SmPr}_{k}^{\text {op }} \rightarrow \operatorname{Mot}_{k}^{\text {aon }} \rightarrow \operatorname{Mot}_{k}$ of the functor $\operatorname{SmPr}_{k}^{\text {op }} \rightarrow \mathbf{M o t}_{k}$. If $H$ is a Weil cohomology theory, then the extension $H: \mathbf{M o t}_{k} \rightarrow \mathbf{g V e c}_{K}$ of Definition 1.7 gives functors $H: \mathbf{M o t}_{k}^{\text {aon }} \rightarrow \mathbf{g V e c}_{K}$ as well.

Note that the all-or-nothing motives $(X, 0)$ for $X$ irreducible are all isomorphic: the maps $0:(X, 0) \rightarrow(Y, 0)$ and vice versa are mutual inverses. This gives an alternative construction for $\mathbf{M o t}_{k}^{\text {aon }}$ :

Definition 3.3. Let $\mathscr{C}$ be a category. Define the category $\mathscr{C} \amalg\{*\}$ whose objects are ob $\mathscr{C} \amalg\{*\}$, and morphisms are given by

$$
\operatorname{Mor}_{\mathscr{C} \amalg\{*\}}(X, Y)= \begin{cases}\operatorname{Mor}_{\mathscr{C}}(X, Y), & X, Y \in \mathrm{ob} \mathscr{C}, \\ \{0\}, & Y=*, \\ \varnothing, & \text { else, }\end{cases}
$$

where $0 \circ f=0$ whenever this makes sense. If $F: \mathscr{C} \rightarrow \mathscr{D}$ is a functor to a category $\mathscr{D}$ with a terminal object *, then there is a unique extension of $F$ to a functor $F: \mathscr{C} \amalg\{*\} \rightarrow \mathscr{D}$ with $F(*)=*$.

Definition 3.4. Let $\mathscr{C}$ be a category. Define the category Coprod $(\mathscr{C})$ of formal finite coproducts in $\mathscr{C}$ whose objects are diagrams $X: I \rightarrow \mathscr{C}$ from a finite discrete category $I$, and morphisms from $X: I \rightarrow \mathscr{C}$ to $Y: J \rightarrow \mathscr{C}$ are given by

$$
\operatorname{Mor}(X, Y)=\prod_{i \in I} \coprod_{j \in J} \operatorname{Mor}\left(X_{i}, Y_{j}\right) .
$$

If $F: \mathscr{C} \rightarrow \mathscr{D}$ is a functor to a category $\mathscr{D}$ with finite coproducts, then there is a unique extension (up to isomorphism) of $F$ to a functor $F: \operatorname{Coprod}(\mathscr{C}) \rightarrow \mathscr{D}$ taking $X: I \rightarrow \mathscr{C}$ to the coproduct $\bigsqcup_{i} F\left(X_{i}\right)$.

If $X: I \rightarrow \mathscr{C}$ is an object of $\operatorname{Coprod}(\mathscr{C})$, then $X$ is the coproduct of the one-object diagrams $X_{i}$, so we may write $X=\bigsqcup_{i} X_{i}$. An initial object of $\operatorname{Coprod}(\mathscr{C})$ is given by the empty diagram $X: \varnothing \rightarrow \mathscr{C}$, and if $*$ is a terminal object of $\mathscr{C}$, then it is also terminal in $\operatorname{Coprod}(\mathscr{C})$.

Example 3.5. We have an equivalence $\operatorname{SmPr} \cong \operatorname{Coprod}\left(\operatorname{SmPrVar}{ }_{k}\right)$, and $\operatorname{Mot}_{k}^{\text {aon }}$ can be defined as $\left(\operatorname{Coprod}\left(\operatorname{SmPrVar}_{k} \amalg\{*\}\right)\right)^{\text {op }}$. In particular, any functor $F: \operatorname{SmPrVar}{ }_{k} \rightarrow \mathscr{D}$ to a category $\mathscr{D}$ with finite coproducts and a terminal object * extends uniquely to a functor $F:\left(\mathbf{M o t}_{k}^{\text {aon }}\right)^{\text {op }} \rightarrow \mathscr{D}$ such that $F(X, 0)=*$ for $X$ irreducible and $F(X, p)=\bigsqcup_{i} F\left(X_{i},\left.p\right|_{X_{i}}\right)$ if the $X_{i}$ are the components of $X$. This gives an alternative method to extend a Weil cohomology $H$ to a functor $H: \mathbf{M o t}_{k}^{\text {aon }} \rightarrow \mathbf{g V e c}_{K}$, cf. Remark 3.2.

Now we are ready to state the additional axioms on our Weil cohomology theory.

Axiom 3.6. Consider the following axioms on a Weil cohomology theory $H$.

(Al) The functor $H: \mathrm{SmPr}_{k}^{\mathrm{op}} \rightarrow \mathbf{g A l g}_{K}$ extends to a compactly supported cohomology functor $H_{\mathrm{c}}: \mathscr{C}^{\mathrm{op}} \rightarrow \mathbf{g V e c}_{K}$ where $\mathscr{C} \subseteq \mathbf{S c h}_{\text {sep, f.t. } / k}$ is the subcategory of proper morphisms. For a closed immersion $Z \hookrightarrow X$ in $\mathscr{C}$ with complementary open $U$, the pullback maps $H_{\mathrm{c}}^{i}(X) \rightarrow H_{\mathrm{c}}^{i}(Z)$ fit into a long exact sequence

$$
\ldots \rightarrow H_{\mathrm{c}}^{i}(U) \rightarrow H_{\mathrm{c}}^{i}(X) \rightarrow H_{\mathrm{c}}^{i}(Z) \rightarrow \ldots
$$

functorial for proper morphisms $f: Y \rightarrow X$ by pulling back $Z$ and $U$. If $X$ is proper, we write $H^{i}(X)$ for $H_{\mathrm{c}}^{i}(X)$. 
(A2) For any $X \in \mathscr{C}$, we have $H_{\mathrm{c}}^{i}(X)=0$ for $i>2 \operatorname{dim} X$. If $X$ is smooth projective of dimension $n$ and $Z \subseteq X$ is a reduced closed subscheme whose irreducible components $Z_{1}, \ldots, Z_{r}$ have dimension $d$, then $H^{2 d}(Z) \cong K^{r}$ and $H^{2 d}(X) \rightarrow H^{2 d}(Z) \cong K^{r}$ is Poincaré dual to the map

$$
\begin{aligned}
K^{r} & \rightarrow H^{2(n-d)}(X) \\
\left(\lambda_{1}, \ldots, \lambda_{r}\right) & \mapsto \sum_{i=1}^{r} \lambda_{i} \operatorname{cl}\left(Z_{i}\right) .
\end{aligned}
$$

(A3) For a cosimplicial all-or-nothing motive $\left(X_{\bullet}, \pi_{\bullet}\right)$, there is a graded $K$-vector space $H^{*}\left(X_{\bullet}, \pi_{\bullet}\right)$ that is computed by a spectral sequence

$$
E_{1}^{p, q}=H^{q}\left(X_{p}, \pi_{p}\right) \Rightarrow H^{p+q}\left(X_{\bullet}, \pi_{\bullet}\right)
$$

that is functorial in $\left(X_{\bullet}, \pi_{\bullet}\right)$, where $E_{1}^{*, q}$ is the alternating face complex on the cosimplicial $K$-vector space $H^{q}\left(X_{\bullet}, \pi_{\bullet}\right)$. If all $\pi_{p}$ are equal to the constant function 1 , then we write $H^{*}\left(X_{\bullet}\right)$ for $H^{*}\left(X_{\bullet}, \pi_{\bullet}\right)$.

(A4) (Cohomological descent for proper hypercoverings) If $X_{\bullet} \rightarrow X$ is a proper hypercovering of a proper $k$-scheme $X$ such that all $X_{n}$ are smooth projective $k$-schemes, then the pullback map

$$
H^{*}(X) \rightarrow H^{*}\left(X_{\bullet}\right)
$$

is an isomorphism.

(A5) If $f_{\bullet}:\left(X_{\bullet}, \alpha_{\bullet}\right) \rightarrow\left(Y_{\bullet}, \beta_{\bullet}\right)$ is a morphism of cosimplicial all-or-nothing motives, then there is a long exact sequence

$$
\ldots \rightarrow H^{i}\left(C_{\bullet}(f)\right) \rightarrow H^{i}\left(X_{\bullet}, \beta_{\bullet}\right) \stackrel{f_{\bullet * *}}{\longrightarrow} H^{i}\left(Y_{\bullet}, \alpha_{\bullet}\right) \rightarrow \ldots
$$

that is functorial in the morphism $f_{\bullet}$, where $C_{\bullet}(f)$ is the mapping cone of cosimplicial all-or-nothing motives, cf. Definition 2.6.

(A6) If $i: Z \hookrightarrow X$ is a closed immersion of proper $k$-schemes with complement $U$, if $X_{\bullet} \rightarrow X$ and $Z_{\bullet} \rightarrow Z$ are proper hypercoverings such that all $X_{n}$ and $Z_{n}$ are smooth projective $k$-schemes, and if $i_{\bullet}: Z_{\bullet} \rightarrow X_{\bullet}$ is a morphism of simplicial schemes fitting in a commutative diagram

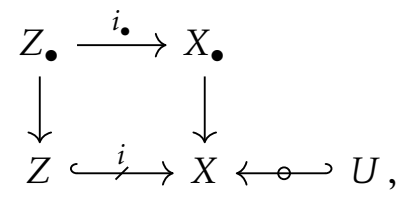

then the isomorphisms $H^{*}(X) \stackrel{\sim}{\rightarrow} H^{*}\left(X_{\bullet}\right)$ and $H^{*}(Z) \stackrel{\sim}{\rightarrow} H^{*}\left(Z_{\bullet}\right)$ of $(\mathrm{A} 4)$ and the long exact sequences of (A1) and (A5) give a commutative diagram

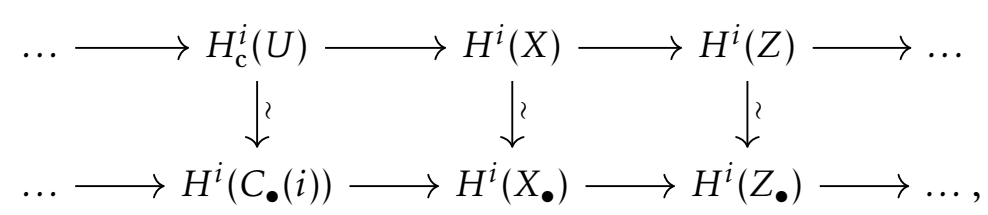


functorial for commutative diagrams

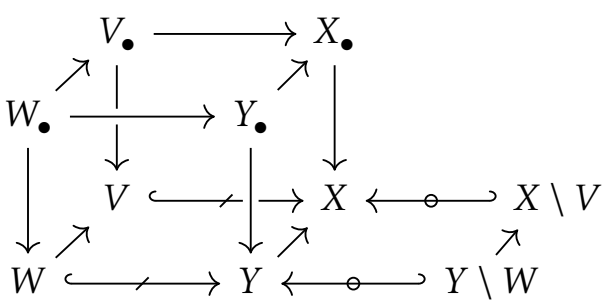

where the bottom squares (but not necessarily the top square) are pullbacks and all vertical maps are proper hypercoverings by smooth projective $k$-schemes.

Example 3.7. If $\ell$ is a prime invertible in $k$, then $\ell$-adic étale cohomology is a Weil cohomology theory by Example 1.2. It satisfies additional axiom (A1) by [SGA4 $4_{\mathrm{III}}$, exp. XVII, 5.1.16]. The vanishing statement in (A2) is given by [SGA4 $4_{\text {III }}$, exp. XVII, cor. 5.2.8.1]. For the computation of $H^{2 d}(Z)$ we may therefore pass to an open and assume $Z$ is smooth, where the result follows from Poincaré duality [SGA4 $4_{\text {III }}$, exp. XVIII, th. 3.2.5]. The final statement of (A2) follows from the definition of the cycle class map [SGA41/2, chap. 4, déf. 2.3.2].

By a (pseudofunctor version of) the universal property of Example 3.5, the pseudofunctor

$$
\begin{aligned}
\left(-, \mathbf{Q}_{\ell}\right): \mathbf{S m P r V a r} & \rightarrow \mathbf{S h v} \\
X & \mapsto\left(X_{\text {pro-êt }}, \mathbf{Q}_{\ell}\right)
\end{aligned}
$$

extends uniquely to a pseudofunctor $F:\left(\mathbf{M o t}_{k}^{\text {aon }}\right)^{\text {op }} \rightarrow \mathbf{S h v}$ that maps * to the pair (Set, 0$)$ and preserves finite coproducts (see Remark 2.8). This corresponds to a fibred and cofibred category $E \rightarrow$ Mot $_{k}^{\text {aon }}$ whose fibre $(X, p)_{\text {pro-ét }}$ over a connected all-or-nothing motive $(X, p)$ is $X_{\text {pro-ét }}$ if $p=1$ and Set if $p=0$, along with a sheaf $\mathscr{F}$ on $\Gamma(E)$ whose restriction to $(X, p)$ is $\mathbf{Q}_{\ell}$ if $p=1$ and 0 if $p=0$.

Given a cosimplicial all-or-nothing motive $\left(X_{\bullet}, \pi_{\bullet}\right): \Delta \rightarrow \operatorname{Mot}_{k}^{\text {aon }}$, the fibre product $E \times{ }_{\mathbf{M o t}_{k}^{\text {an }}} \Delta \rightarrow \Delta$ is a simplicial topos [SGA4 $4_{\mathrm{II}}$, exp. $\mathrm{V}^{\text {bis }}, 1.2 .5$ ], which we will denote by $\left(X_{\bullet}, \pi_{\bullet}\right)_{\text {pro-ét }}$.

The sheaf $\mathscr{F}$ on $\Gamma(E)$ pulls back to $\Gamma\left(\left(X_{\bullet}, \pi_{\bullet}\right)_{\text {pro-ét }}\right)$, and the spectral sequence of Remark 2.4 then reads

$$
E_{1}^{p, q}=H^{q}\left(\left(X_{p}, \pi_{p}\right)_{\text {pro-ét }}, \mathscr{F}\right) \Rightarrow H^{p+q}\left(\left(X_{\bullet}, \pi_{\bullet}\right), \mathscr{F}\right) .
$$

For any all-or-nothing motive $(X, \pi)$, we have $H^{q}\left((X, \pi)_{\text {pro-ét }}, \mathscr{F}\right)=H^{q}(X, \pi)$, where the right hand side is defined by Remark 3.2. This gives the required spectral sequence of (A3). Moreover, (A5) holds by Lemma 2.9 , since all (pseudo)functors involved preserve terminal objects and finite coproducts, hence preserve the construction of the mapping cone. Cohomological descent (A4) follows from [SGA4 ${ }_{\text {II }}$, exp. $\mathrm{V}^{\text {bis }}$, prop. 4.3.2, th. 3.3.3, and prop. 2.5.7].

Finally, in the situation of (A6), the functoriality statement in Lemma 2.9 immediately reduces us to the case of the trivial hypercoverings $X_{\bullet} \rightarrow X$ and $Z_{\bullet} \rightarrow Z$ given by the constant simplicial schemes $X_{n}=X$ and $Z_{n}=Z$. (This does not preserve the hypothesis that each $X_{n}$ and $Z_{n}$ is smooth projective, but étale cohomology is defined and has all the desired properties also for simplicial objects in $\mathbf{S c h}_{\text {sep, f.t./k. }}$.

In fact, since $i_{*}$ is exact, the same goes for $\Gamma(i)_{*}: \mathbf{A b}\left(Z_{\bullet}\right) \rightarrow \mathbf{A} \mathbf{b}\left(X_{\bullet}\right)$, so we may replace the morphism $\left(Z_{\bullet}, \mathbf{Q}_{\ell, \bullet}\right) \rightarrow\left(X_{\bullet}, \mathbf{Q}_{\ell, \bullet}\right)$ by $\left(X_{\bullet}, i_{*} \mathbf{Q}_{\ell, \bullet}\right) \rightarrow\left(X_{\bullet}, \mathbf{Q}_{\ell, \bullet}\right)$ using the functoriality assertion of Lemma 2.9. An injective resolution

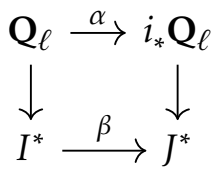




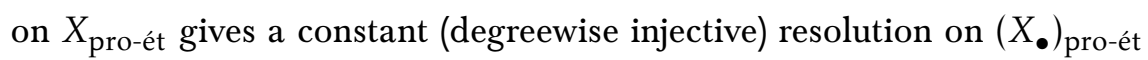

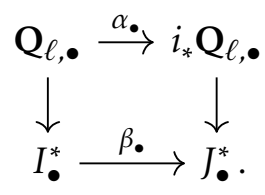

This gives a commutative diagram with exact rows

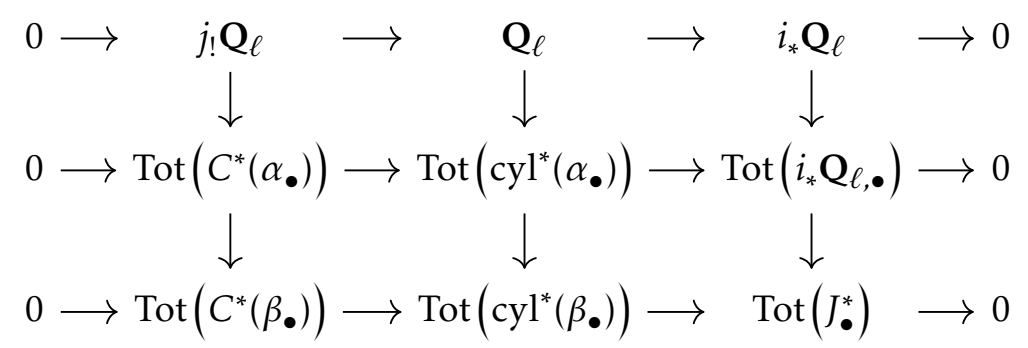

of complexes on $X_{\text {pro-ét }}$, whose vertical maps are quasi-isomorphisms, where $C^{*}$ and cyl ${ }^{*}$ denote the dual constructions to Definition 2.6, applied to the rows of (3.3) viewed as morphisms of cosimplicial objects in $\mathbf{C h}\left(X_{\text {pro-ét }}\right)$. The terms in the bottom row of (3.4) are injective, so the long exact sequence of (Al) is computed by

$$
0 \rightarrow \operatorname{Tot}\left(\Gamma\left(X, C^{*}\left(\beta_{\bullet}\right)\right)\right) \rightarrow \operatorname{Tot}\left(\Gamma\left(X, \operatorname{cyl}^{*}\left(\beta_{\bullet}\right)\right)\right) \rightarrow \operatorname{Tot}\left(\Gamma\left(X, J_{\bullet}^{*}\right)\right) \rightarrow 0 .
$$

This agrees with the sequence (2.3) for the constant resolution (3.3), giving the commutative diagram (3.1). This construction is functorial for commutative diagrams (3.2) since pullback along $Y \rightarrow X$ preserves the short exact sequence $0 \rightarrow j_{!} \mathbf{Q}_{\ell} \rightarrow \mathbf{Q}_{\ell} \rightarrow i_{*} \mathbf{Q}_{\ell} \rightarrow 0$ and by the functoriality statement in Lemma 2.9.

Example 3.8. If $k$ is a perfect field of positive characteristic $p$ with Witt ring $W(k)$ with field of fractions $K$, then crystalline cohomology $H_{\text {cris }}^{i}(X / K)$ is a Weil cohomology theory by Example 1.3. Axiom (A1) is provided by rigid cohomology with compact support [Ber86, 3.1(iii)], and (A4) is [Tsu03, Theorem 4.5.1]. The proofs of axioms (A2) (using Poincaré duality for rigid cohomology of smooth varieties [Ber97]), (A3), (A5), and (A6) (using Le Stum's site theoretic definition of rigid cohomology [LeS11]) are analogous to Example 3.7.

Remark 3.9. The above axioms are exactly what we need, but possibly not the most natural choice. It's likely that our axioms (or an alternative set of sufficient axioms) can be deduced from Cisinski-Déglise's axioms for a mixed Weil cohomology theory [CD12]. Somewhat surprisingly, cohomological descent for proper hypercoverings (A4) is indeed always satisfied [CD19, Corollary 17.2.6].

\section{Independence of Weil cohomology theory}

As in Section 1, we assume that $k$ is an algebraically closed field, and we fix an adequate equivalence relation $\sim$ that is finer than homological equivalence for any Weil cohomology theory. From now on, we will fix Weil cohomology theories $H$ and $\mathcal{H}$ (see Definition 1.1) with coefficient fields $K$ and $\mathcal{K}$ respectively. We will always assume that $H$ and $\mathcal{H}$ satisfy the additional properties (A1-6) of Axiom 3.6.

Definition 4.1. Let $X$ and $Y$ be separated $k$-schemes of finite type. Consider the following statements on independence of Weil cohomology theory:

$\operatorname{Dim}_{\mathrm{c}}(X): \quad$ for each $i$, the dimensions of $H_{\mathrm{c}}^{i}(X)$ and $\mathcal{H}_{\mathrm{c}}^{i}(X)$ agree.

$\operatorname{Rk}_{\mathrm{c}}(X, Y): \quad$ for any proper morphism $f: Y \rightarrow X$ and any $i$, the ranks of $f^{*}$ on $H_{\mathrm{c}}^{i}$ and $\mathcal{H}_{\mathrm{c}}^{i}$ agree.

If $X$ and $Y$ are smooth projective, we further consider:

$\mathrm{Cl}(X): \quad$ the kernels of the cycle class maps cl: $\mathrm{CH}_{\mathbf{Q}}^{*}(X) \rightarrow H^{*}(X)$ and cl: $\mathrm{CH}_{\mathbf{Q}}^{*}(X) \rightarrow \mathcal{H}^{*}(X)$ agree. 
$\operatorname{Rk}^{r}(X, Y): \quad$ for any $\alpha \in \operatorname{Corr}^{r}(X, Y)$ and any $i$, the ranks of $\alpha_{*}$ on $H^{i}$ and $\mathcal{H}^{i}$ agree.

$\operatorname{Rk}(X, Y): \quad \operatorname{Rk}^{0}(X, Y)$ holds.

$\operatorname{Rk}^{*}(X, Y): \quad \operatorname{Rk}^{r}(X, Y)$ holds for all $r \in \mathbf{Z}$.

$\operatorname{Kün}(X): \quad$ for each $i$, there exists a cycle $p \in \operatorname{Corr}(X, X)$ inducing the $i^{\text {th }}$ Künneth projector on both $H^{*}$ and $\mathcal{H}^{*}$.

The reliance of these properties on the chosen Weil cohomology theories $H$ and $\mathcal{H}$ will be implicit, and we will make no further mention of it.

Remark 4.2. For Chow motives $M=(X, p, m), N=(Y, q, n)$ one can also define similar statements $\operatorname{Dim}(M)$ and $\operatorname{Rk}(M, N)$. But these are already implied by $\operatorname{Rk}(X, X)$ and $\operatorname{Rk}^{*}(X, Y)$ respectively: the dimension of $H^{i}(M)$ is the rank of $p_{*}: H^{i}(X) \rightarrow H^{i}(X)$, and the rank of $\alpha_{*}: H^{i}(M) \rightarrow H^{i}(N)$ is the rank of $(q \alpha p)_{*}: H^{i+2 m}(X) \rightarrow H^{i+2 n}(Y)$.

Remark 4.3. For $\operatorname{Kün}(X)$, note that such a cycle $p$ need not be a projector in $\operatorname{Corr}(X, X)$. We only know that the cycle class map sends it to a projector in both $\operatorname{End}\left(H^{*}(X)\right)$ and $\operatorname{End}\left(\mathcal{H}^{*}(X)\right)$.

Remark 4.4. If $k=\overline{\mathbf{F}}_{p}$ and $X$ is smooth proper, then we know $\operatorname{Dim}_{\mathrm{c}}(X)$ (for any Weil cohomology theories $H, \mathcal{H})$, because the dimension can be read off from the zeta function. On the other hand, $\operatorname{Rk}_{\mathrm{c}}(X, Y)$ is still unknown even when $X$ and $Y$ are smooth and projective.

We also know $\operatorname{Kün}(X)$ for $X$ smooth projective over $\overline{\mathbf{F}}_{p}$, by [KM74]. Hence by Corollary 1.15, the characteristic polynomial of $\alpha \in \operatorname{Corr}(X, X)$ is independent of the Weil cohomology theory. In particular, if $\alpha=p$ is a projector, this implies that $\operatorname{dim} H^{i}(X, p, 0)$ is independent of $H$.

Remark 4.5. If char $k=0$, then for all known cohomology theories $H$ and $\mathcal{H}$, the statements $\mathrm{Cl}(X)$ and $\operatorname{Rk}^{*}(X, Y)$ for $X$ and $Y$ smooth projective, as well as $\operatorname{Dim}_{\mathrm{c}}(X)$ and $\mathrm{Rk}_{\mathrm{c}}(X, Y)$ for $X$ and $Y$ separated and of finite type over $k$ are known. On the other hand, $\operatorname{Kün}(X)$ is still open, even for (smooth projective) varieties over C.

Theorem 4.6. Let $k=\overline{\mathbf{F}}_{p}$. Then the following are equivalent:

(1) For all smooth projective $k$-schemes $X$, we have $\mathrm{Cl}(X)$.

(2a) For all smooth projective $k$-schemes $X$ and $Y$, we have $\operatorname{Rk}^{*}(X, Y)$;

(2b) For all smooth projective $k$-schemes $X$ and $Y$, we have $\operatorname{Rk}(X, Y)$;

(2c) For all smooth projective $k$-schemes $X$, we have $\operatorname{Rk}(X, X)$;

(3a) For all separated finite type $k$-schemes $X$ and $Y$, we have $\operatorname{Dim}_{\mathrm{c}}(X)$ and $\operatorname{Rk}_{\mathrm{c}}(X, Y)$;

(3b) For all smooth, quasi-projective $k$-schemes $X$, we have $\operatorname{Dim}_{c}(X)$.

The outline of the rest of the article is as follows. In each of the following sections, we will prove one of the implications, often in a more refined version. We will prove the implications in the following cyclic order:

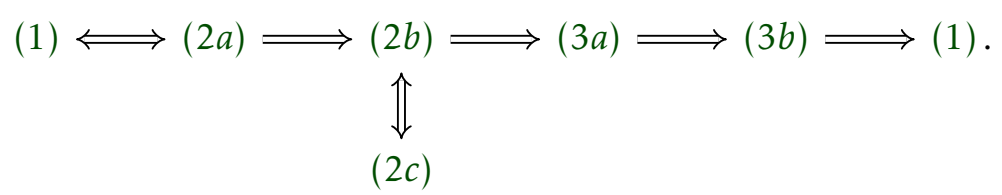

Implications $(2 a) \Rightarrow(2 b) \Rightarrow(2 c)$ and $(3 a) \Rightarrow(3 b)$ are trivial. For $(2 c) \Rightarrow(2 b)$, recall that

$$
\operatorname{Corr}(X, Y)=\operatorname{Hom}_{\mathbf{M o t}_{k}}((X, \mathrm{id}, 0),(Y, \mathrm{id}, 0)) \text {, }
$$

so $\operatorname{Rk}(X \amalg Y, X \amalg Y)$ implies $\operatorname{Rk}(X, Y)$ since $X \amalg Y$ is the biproduct in $\operatorname{Mot}_{k}$ (see Remark 1.12), the functors $H: \mathbf{M o t}_{k} \rightarrow \mathbf{g V e c}_{K}$ and $\mathcal{H}: \mathbf{M o t}_{k} \rightarrow \mathbf{g V e c}_{\mathcal{K}}$ preserve biproducts, and the rank of a block matrix $\left(\begin{array}{ll}0 & 0 \\ A & 0\end{array}\right)$ is the rank of $A$. 
The implications $(1) \Leftrightarrow(2 a),(2 b) \Rightarrow(3 a)$, and $(3 b) \Rightarrow(1)$ will be the contents of the following three sections (Section 5, Section 6, Section 7) respectively.

Remark 4.7. The proof of $(1) \Rightarrow(2 a)$ relies on $\operatorname{Kün}(X)$, which currently is known only when $k=\overline{\mathbf{F}}_{p}$. Implication $(2 b) \Rightarrow(3 a)$ uses the Weil conjectures and a hypercovering argument, so also does not generalise to other fields (but see Remark 4.8). The implication $(3 b) \Rightarrow(1)$ holds over an arbitrary algebraically closed field.

Remark 4.8. Suppose $H$ and $\mathcal{H}$ are given by étale cohomology. If $S$ is an irreducible scheme, $\bar{s}$ and $\bar{\eta}$ are a geometric point and a geometric generic point, $\ell$ is a prime invertible on $S$, and $X \rightarrow S$ is a smooth proper morphism of schemes, then the smooth and proper base change theorems give isomorphisms

$$
\text { sp: } H^{i}\left(X_{\bar{s}}, \mathbf{Q}_{\ell}\right) \cong H^{i}\left(X_{\bar{\eta}}, \mathbf{Q}_{\ell}\right) \text {; }
$$

see for example [SGA4 $4_{\text {III }}$, exp. XVI, cor. 2.2]. Using a standard spreading out argument, this shows that statements (1) and (2abc) for $\overline{\mathbf{F}}_{p}$ for one prime $p$ (resp. every prime $p$ ) imply the same result for any algebraically closed field $k$ of characteristic $p$ (resp. any algebraically closed field $k$ ).

Moreover, for étale cohomology the argument in $(2 b) \Rightarrow(3 a)$ can be refined to deduce (3a) over an arbitrary algebraically closed field $k$ from $(2 \mathrm{~b})$ over $\overline{\mathbf{F}}_{p}$, again using spreading out and the specialisation isomorphism (4.1). The argument for $(3 b) \Rightarrow(1)$ works over any algebraically closed field, so we see that the case $k=\overline{\mathbf{F}}_{p}$ is the essential one.

Remark 4.9. If $X$ is smooth projective over $k$ and $\sim$ is rational equivalence, we get a ring isomorphism

$$
\text { ch: } K_{\mathbf{Q}}(X) \rightarrow \mathrm{CH}_{\mathbf{Q}}^{*}(X) \text {. }
$$

Thus, $\mathrm{Cl}(X)$ is equivalent to the following statement:

$\mathrm{Cl}^{\prime}(X): \quad$ the kernels of the Chern character maps ch: $K_{\mathbf{Q}}(X) \rightarrow H^{*}(X)$ and ch: $K_{\mathbf{Q}}(X) \rightarrow \mathcal{H}^{*}(X)$ agree.

To study the vanishing of $\operatorname{ch}_{H}(\alpha)$ for $\alpha \in K_{\mathbf{Q}}(X)$, the splitting principle plus injectivity of pullbacks for dominant maps [Kle68, Proposition 1.2.4] reduces us to the case where $\alpha$ is in the subring of $K_{\mathbf{Q}}(X)$ generated by classes of the form $[\mathscr{L}]$ for $\mathscr{L}$ a line bundle on $X$. Under the isomorphism (4.2), this corresponds to the subalgebra of $\mathrm{CH}_{\mathbf{Q}}^{*}(X)$ generated by divisors (but note that $\operatorname{ch}([D]) \neq[\mathcal{O}( \pm D)]$ ).

Although $\mathrm{Cl}(X)$ is known for divisors, it seems that this cannot be used to deduce the statement in general.

\section{Cycle classes and ranks}

In this section, $k$ is an arbitrary algebraically closed field. However, we will soon assume that Kün holds, which is currently only known for $k=\overline{\mathbf{F}}_{p}[\mathrm{KM} 74]$.

Theorem 5.1. Let $X$ and $Y$ be smooth projective $k$-schemes.

(1) Assume $\mathrm{Rk}^{*}(\operatorname{Spec} k, X)$. Then $\mathrm{Cl}(X)$ holds.

(2) Assume $\operatorname{Kün}(X), \operatorname{Kün}(Y)$, and $\operatorname{Cl}\left((X \times Y)^{n}\right)$ for all $n$. Then $\operatorname{Rk}^{*}(X, Y)$ holds.

Proof. We have $\operatorname{Corr}^{r}(\operatorname{Spec} k, X)=\mathrm{CH}_{\mathbf{Q}}^{r}(X)$, and a cycle $\alpha \in \mathrm{CH}_{\mathbf{Q}}^{r}(X)$ maps to zero under the cycle class map cl: $\mathrm{CH}_{\mathbf{Q}}^{r}(X) \rightarrow H^{2 r}(X)$ if and only if $\alpha_{*}: H^{*}(\operatorname{Spec} k) \rightarrow H^{*}(X)$ is zero (and similarly for $\mathcal{H}^{*}$ ); see Lemma 1.13. Now (1) follows from the assumption that $H^{*}(\operatorname{Spec} k)$ is 1 -dimensional, so the only possibilities for the rank of $\alpha_{*}$ are 0 and 1 , corresponding to $\alpha_{*}=0$ and $\alpha_{*} \neq 0$ respectively.

For (2), let $i$ and $r$ be given, and let $p \in \operatorname{Corr}(X, X)$ (resp. $q \in \operatorname{Corr}(Y, Y))$ be an element acting on $H^{*}$ and $\mathcal{H}^{*}$ as the $i^{\text {th }}$ (resp. $\left.i+2 r^{\text {th }}\right)$ Künneth projector. For $\alpha \in \operatorname{Corr}^{r}(X, Y)$, we get an induced element

$$
q \circ \alpha \circ p \in \operatorname{Corr}^{r}(X, Y) \text {. }
$$


Moreover, the map $(q \alpha p)_{*}: H^{i}(X) \rightarrow H^{i+2 r}(Y)$ agrees with the map induced by $\alpha$ (and the same holds for $\mathcal{H}^{i}(X) \rightarrow \mathcal{H}^{i+2 r}(Y)$ ). Denote this map by $\alpha_{i}$ (on both $H^{i}$ and $\mathcal{H}^{i}$ ). First assume $i$ is even, and consider the induced maps

$$
\bigwedge^{j}(q \alpha p): \wedge^{j} X \vdash \wedge^{j} Y
$$

for various $j$. By Remark 1.11, we have a decomposition

$$
H^{*}\left(\wedge^{j} X\right)=\bigoplus_{a+b=j} \wedge^{a} H^{\text {even }}(X) \otimes S^{b} H^{\text {odd }}(X) .
$$

The map $H^{*}\left(\bigwedge^{j} X\right) \rightarrow H^{*}\left(\bigwedge^{j} Y\right)$ induced by $\bigwedge^{j}(q \alpha p)$ is $\bigwedge^{j} \alpha_{i}$ on $\bigwedge^{j} H^{i}(X)$, and 0 on all other components of $H^{*}\left(\bigwedge^{j} X\right)$. In particular, it is nonzero if and only if $j \leq \operatorname{rk}\left(\left.\alpha_{i}\right|_{H}\right)$. Similarly, the map on $\mathcal{H}^{*}(X)$ induced by $\wedge^{j}(q \alpha p)$ is nonzero on $\mathcal{H}^{*}(X)$ if and only if $j \leq \operatorname{rk}\left(\left.\alpha_{i}\right|_{\mathcal{H}}\right)$. Thus, the rank of $\alpha_{i}$ only depends on the vanishing or nonvanishing of the cycles $\bigwedge^{j}(q \alpha p)$ under the cycle class map, by Lemma 1.13. But we assumed that the kernels of the cycle class maps are the same for $H^{*}\left((X \times Y)^{j}\right)$ and $\mathcal{H}^{*}\left((X \times Y)^{j}\right)$. This proves the claim if $i$ is even. If $i$ is odd, we use $S^{j}$ instead of $\bigwedge^{j}$ (see Remark 1.11).

Corollary 5.2. Let $k$ be an algebraically closed field such that $\mathrm{Kün}(X)$ holds for all smooth projective $k$-schemes $X$ (e.g. $\left.k=\overline{\mathbf{F}}_{p}[\mathrm{KM} 74]\right)$. Then the following are equivalent:

(1) $\mathrm{Cl}(X)$ holds for all smooth projective $k$-schemes $X$;

(2) $\operatorname{Rk}^{*}(X, Y)$ holds for all smooth projective $k$-schemes $X$ and $Y$.

\section{Ranks and dimensions}

In this section, $k$ will denote an arbitrary field. The main result of this section (Theorem 6.6) assumes that $k=\overline{\mathbf{F}}_{p}$, because its proof relies on the Weil conjectures. The idea is to use alterations to produce smooth hypercoverings that compute the cohomology of arbitrary separated finite type $k$-schemes.

Lemma 6.1. Let $X$ and $Y$ be separated finite type $k$-schemes, and $f: Y \rightarrow X$ a morphism. Then there exist proper $k$-schemes $\bar{X}, \bar{Y}$ along with dense open immersions $X \rightarrow \bar{X}$ and $Y \rightarrow \bar{Y}$ and a morphism $\bar{f}: \bar{Y} \rightarrow \bar{X}$ such that the diagram

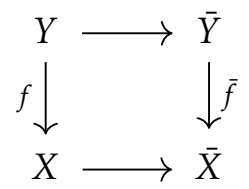

commutes. If $f$ is proper, then $Y=\bar{f}^{-1}(X)$.

Proof. Let $X \rightarrow \bar{X}$ be a Nagata compactification [Nag62]. Replacing $\bar{X}$ by the closure of $X$ in $\bar{X}$, we may assume that $X$ is dense in $\bar{X}$. Let $\bar{Y}$ be a relative Nagata compactification of $Y \rightarrow \bar{X}$ [Nag63]. Again, we may assume that $Y$ is dense in $\bar{Y}$. Then $\bar{Y}$ is proper over $\bar{X}$, hence proper over $k$ since $\bar{X}$ is. This proves the first statement. The second statement follows because the scheme theoretic image of the morphism of proper $X$-schemes $Y \rightarrow \bar{f}^{-1}(X)$ is closed. Since $Y$ is also dense in $\bar{f}^{-1}(X)$ (in fact, in $\bar{Y}$ ), this forces equality.

Lemma 6.2. Let $(\mathscr{J}, \leq)$ be a poset (viewed as category) such that for every $i \in \mathscr{J}$ there are only finitely many $j \in \mathscr{J}$ with $j \leq i$. Let $D_{0}: \mathscr{J}^{\mathrm{op}} \rightarrow \mathbf{S c h}_{k}$ be a diagram of separated finite type $k$-schemes. Then there exists a diagram $D_{1}: \mathscr{J}^{\mathrm{op}} \rightarrow \mathbf{S c h}_{k}$ and a map $D_{1} \rightarrow D_{0}$ such that

(1) each $D_{1}(i) \rightarrow D_{0}(i)$ is proper and surjective, and

(2) each $D_{1}(i)$ is quasi-projective over $k$ and regular. 
Proof. We will construct $D_{1} \rightarrow D_{0}$ as a functor $D:(\mathscr{J} \times[1])^{\text {op }} \rightarrow \mathbf{S c h}_{k}$ with the desired properties. For $i \in \mathscr{J}$ and $J \subseteq \mathscr{J}$, write $J_{<i}=\{j \in J \mid j<i\}$ and $J_{\leq i}=\{j \in J \mid j \leq i\}$, and set

$$
C_{J, i}:=\left(\mathscr{J}_{\leq i} \times\{0\}\right) \cup\left(J_{\leq i} \times\{1\}\right) \subseteq \mathscr{J}_{\leq i} \times[1] .
$$

If $D$ is defined on $(J \times[1])^{\mathrm{op}}$ and $i \in \mathscr{J}$ is arbitrary, we write

$$
L_{J}(i):=\lim _{C_{J, i}^{\mathrm{op}}} D
$$

Since this is a finite limit, it exists in $\mathbf{S c h}_{k}$ and is separated and of finite type. We always have $L_{\varnothing}=D_{0}$, and we have $L_{\mathscr{J}_{\leq i}}(i)=D_{1}(i)$ and write $L(i):=L_{\mathscr{J}_{<i}}(i)$ when these are defined.

By induction on the size of the finite poset $\mathscr{J}_{<i}$, we construct $D_{1}(i)$ as a cone over the restriction of $D$ to $C_{\mathscr{J}_{<i}, i}=\left(\mathscr{J}_{\leq i} \times[1]\right) \backslash(i, 1)$, such that moreover the natural map $D_{1}(i) \rightarrow L(i)$ is proper and surjective. If $i$ is minimal (i.e. $\mathscr{J}_{<i}=\varnothing$ ), take $D_{1}(i) \rightarrow D_{0}(i)$ an alteration [dJo96, Theorem 4.1]. Since $L(i)=L_{\varnothing}(i)=D_{0}(i)$, the map $D_{1}(i) \rightarrow L(i)$ is proper and surjective by construction.

Now take $i \in \mathscr{J}$ arbitrary, and assume $D_{1}(j)$ has been defined and $D_{1}(j) \rightarrow L(j)$ is proper and surjective for all $j$ with $\left|\mathscr{J}_{<j}\right|<\left|\mathscr{J}_{<i}\right|$; in particular for all $j \in \mathscr{J}_{<i}$. Then $L(i)$ is defined, and we take $D_{1}(i) \rightarrow L(i)$ any alteration [dJo96]. The required functoriality $D_{1}(i) \rightarrow D_{1}(j)$ for $j<i$ comes from the fact that $L(i)$ is a cone over the restriction of $D$ to $C_{\mathscr{J}_{<i}, i}$.

If $J \subseteq \mathscr{J}_{<i}$ is downward closed and $j \in J$ is a maximal element, then $J \backslash\{j\}$ is downward closed, and we have

$$
L_{J}(i)=L_{J \backslash\{j\}}(i) \underset{L(j)}{\times} D_{1}(j) ;
$$

in particular $L_{J}(i) \rightarrow L_{J \backslash\{j\}}(i)$ is proper and surjective. By induction on $|J|$, we conclude that $L(i) \rightarrow D_{0}(i)$ and hence $D_{1}(i) \rightarrow D_{0}(i)$ is proper and surjective. Finally, $D_{1}(i)$ is a regular, quasi-projective $k$-scheme by construction.

We note that the proof above uses nothing special about schemes, and it generalises without difficulty to the categorical setting of [SGA4 $4_{\mathrm{II}}$, exp. $\left.\mathrm{V}^{\mathrm{bis}}, 5.1 .4\right]$.

Corollary 6.3. Let $(\mathscr{J}, \leq)$ be a poset such that for every $i \in \mathscr{J}$ there are only finitely many $j \in \mathscr{J}$ with $j \leq i$. Let $D: \mathscr{J}^{\mathrm{op}} \rightarrow \mathbf{S c h}_{k}$ be a diagram of separated finite type $k$-schemes. Then there exists a diagram $D_{\bullet}: \mathscr{J}^{\mathrm{op}} \times \Delta_{+}^{\mathrm{oP}} \rightarrow \mathbf{S c h}_{k}$ such that the following hold.

(1) $D_{-1}=D$;

(2) For each $i \in \mathscr{J}$, the diagram $D_{\bullet}(i)$ is a proper hypercovering of $D(i)$;

(3) For each $i \in \mathscr{J}$ and each $n \in \mathbf{Z}_{\geq 0}$, the scheme $D_{n}(i)$ is quasi-projective over $k$ and regular.

Proof. Apply the procedure of [SGA4 ${ }_{\mathrm{II}}$, exp. $\left.\mathrm{V}^{\mathrm{bis}}, 5.1 .4-5.1 .7,5.2 .4\right]$ (see also [Del74b, 6.2.8]), replacing $k$-schemes by $\mathscr{J}$-indexed diagrams of $k$-schemes, and resolution of singularities (or alterations) by Lemma 6.2 .

Lemma 6.4. Let $0 \rightarrow A^{\bullet} \rightarrow B^{\bullet} \rightarrow C^{\bullet} \rightarrow 0$ be a short exact sequence of chain complexes of finite dimensional vector spaces. Then

$$
\operatorname{rk}\left(\delta^{i}: H^{i}\left(C^{\bullet}\right) \rightarrow H^{i+1}\left(A^{\bullet}\right)\right)=\operatorname{rk}\left(d_{B}^{i}\right)-\operatorname{rk}\left(d_{A}^{i}\right)-\operatorname{rk}\left(d_{C}^{i}\right)
$$

Proof. All ranks in question only depend on the stupid truncations $\sigma_{\geq i} \sigma_{\leq i+1}$ of the complexes. For $d_{A}^{i}, d_{B}^{i}$, and $d_{C}^{i}$ this is clear, and for $\delta^{i}$ this follows because of the factorisation

$$
H^{i}\left(\sigma_{\geq i} C^{\bullet}\right) \rightarrow H^{i}\left(C^{\bullet}\right) \stackrel{\delta^{i}}{\rightarrow} H^{i+1}\left(A^{\bullet}\right) \hookrightarrow H^{i+1}\left(\sigma_{\leq i+1} A^{\bullet}\right),
$$


noting that precomposing by surjections and postcomposing by injections does not alter ranks. Now the snake lemma gives a long exact sequence

$$
0 \rightarrow \operatorname{ker} d_{A}^{i} \rightarrow \operatorname{ker} d_{B}^{i} \rightarrow \operatorname{ker} d_{C}^{i} \stackrel{\delta^{i}}{\rightarrow} \operatorname{coker} d_{A}^{i} \rightarrow \operatorname{coker} d_{B}^{i} \rightarrow \operatorname{coker} d_{C}^{i} \rightarrow 0 .
$$

Additivity of dimension in short exact sequences gives

$$
\operatorname{dim}\left(\operatorname{ker} d_{A}^{i}\right)-\operatorname{dim}\left(\operatorname{ker} d_{B}^{i}\right)+\operatorname{dim}\left(\operatorname{ker} d_{C}^{i}\right)-\operatorname{rk}\left(\delta^{i}\right)=0 .
$$

On the other hand, exactness of $0 \rightarrow A^{\bullet} \rightarrow B^{\bullet} \rightarrow C^{\bullet} \rightarrow 0$ gives

$$
\operatorname{dim} A^{i}-\operatorname{dim} B^{i}+\operatorname{dim} C^{i}=0 .
$$

Subtracting (6.1) from (6.2) gives the result.

Corollary 6.5. Let $f: A^{\bullet} \rightarrow B^{\bullet}$ be a morphism of chain complexes of finite dimensional vector spaces. Then

$$
\operatorname{rk}\left(H^{i}(f)\right)=\operatorname{rk}\left(\left(\begin{array}{cc}
d_{A}^{i} & 0 \\
f^{i} & -d_{B}^{i-1}
\end{array}\right): A^{i} \oplus B^{i-1} \rightarrow A^{i+1} \oplus B^{i}\right)-\operatorname{rk}\left(d_{A}^{i}\right)-\operatorname{rk}\left(d_{B}^{i-1}\right) .
$$

Proof. Apply Lemma 6.4 to the short exact sequence

$$
0 \rightarrow B^{\bullet}[-1] \rightarrow C^{\bullet}(f) \rightarrow A^{\bullet} \rightarrow 0,
$$

noting that the boundary homomorphism of this sequence is $H^{i}(f)$.

Theorem 6.6. Assume $k=\overline{\mathbf{F}}_{p}$. If $\operatorname{Rk}(X, Y)$ holds for all smooth projective $k$-schemes $X$ and $Y$, then

(1) $\operatorname{Dim}_{\mathrm{c}}(X)$ holds for every separated finite type $k$-scheme $X$;

(2) $\operatorname{Rk}_{\mathrm{c}}(X, Y)$ holds for all separated finite type $k$-schemes $X$ and $Y$.

Proof. Let $X$ and $Y$ be separated $k$-schemes of finite type, and let $f: Y \rightarrow X$ be a proper morphism. Choose a commutative diagram as in Lemma 6.1

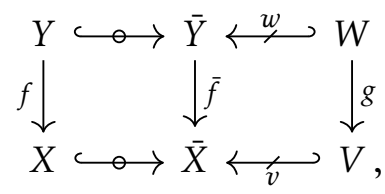

where $\bar{X}$ and $\bar{Y}$ are compatible compactifications of $X$ and $Y$ respectively such that $X$ (resp. $Y$ ) is dense in $\bar{X}$ (resp. $\bar{Y}$ ) with complement $V$ (resp. $W$ ), and $g$ denotes $\left.\bar{f}\right|_{W}$. Applying Corollary 6.3 to the right hand square of (6.3), we get a commutative diagram

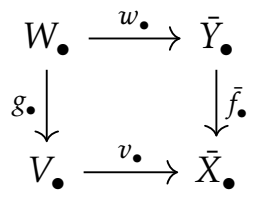

of augmented simplicial schemes such that each $W_{i}$ is smooth projective for $i \geq 0$ and $W_{\bullet}$ is a proper hypercover of $W_{-1}=W$, and similarly for $V, \bar{X}$, and $\bar{Y}$.

By Axiom 3.6 (A1), (A5), and (A6), we get a commutative square of long exact sequences

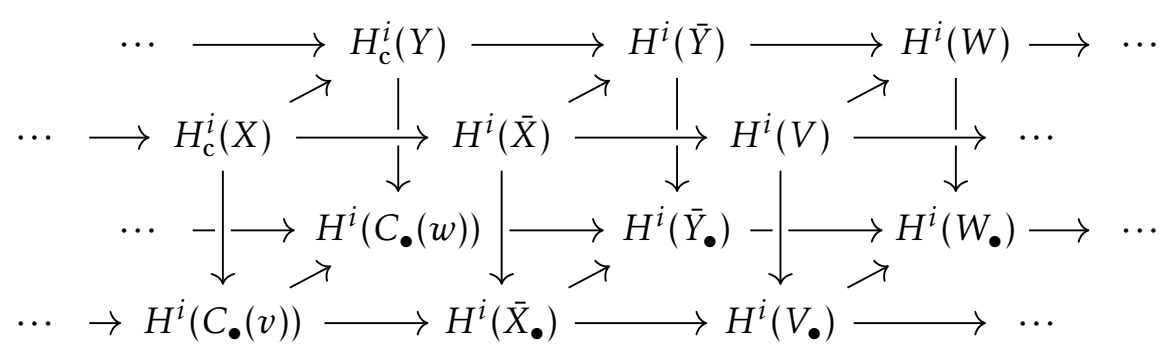


whose vertical maps are isomorphisms. Thus $H_{\mathrm{c}}^{i}(X)$ is computed by $H^{i}\left(C_{\bullet}(v)\right)$, which by (A3) is computed by the hypercohomology spectral sequence

$$
E_{1}^{p, q}=H^{q}\left(C_{p}(v)\right) \Rightarrow H^{p+q}\left(C_{\bullet}(v)\right) .
$$

If $\bar{X}_{p}$ and $V_{p}$ are defined over some finite field $k_{0}$ for all $p \leq i+1$, then the computation of $H^{i}\left(C_{\bullet}(v)\right)$ only involves maps between cohomology groups of smooth projective varieties defined over $k_{0}$. Moreover, the action of the $\left|k_{0}\right|$-power geometric Frobenius (as an algebraic cycle) on $H^{q}\left(C_{p}(v)\right)$ is pure of weight $q$ [KM74, Corollary $1(2)]$ (see also Corollary 1.15).

Since $\operatorname{Ext}_{K[x]}^{i}(M, N)=0$ for all $i$ and for all $K[x]$-modules $M$ and $N$ that are pure of different weights, the spectral sequence (6.6) degenerates on the $E_{2}$ page and the filtration on $E_{\infty}$ canonically splits:

$$
H_{\mathrm{c}}^{i}(X)=H^{i}\left(C_{\bullet}(v)\right) \cong \bigoplus_{p+q=i} E_{2}^{p, q}
$$

where $E_{2}^{p, q}$ is given by

$$
E_{2}^{p, q}=\frac{\operatorname{ker}\left(d^{p}: H^{q}\left(C_{p}(v)\right) \rightarrow H^{q}\left(C_{p+1}(v)\right)\right)}{\operatorname{im}\left(d^{p-1}: H^{q}\left(C_{p-1}(v)\right) \rightarrow H^{q}\left(C_{p}(v)\right)\right)}
$$

A dimension count gives

$$
\operatorname{dim} E_{2}^{p, q}=\operatorname{dim}\left(H^{q}\left(C_{p}(v)\right)\right)-\operatorname{rk}\left(d^{p}\right)-\operatorname{rk}\left(d^{p-1}\right) .
$$

By assumption and by Remark 4.2, each of these numbers is independent of the Weil cohomology theory $H$. Then the same holds for $\operatorname{dim} E_{2}^{p, q}$ and therefore also for $\operatorname{dim} H_{\mathrm{c}}^{i}(X)$, which proves (1) for $X$.

By (A3), the natural map $f_{\bullet}: C_{\bullet}(v) \rightarrow C_{\bullet}(w)$ of cosimplicial all-or-nothing motives induces a morphism of spectral sequences

$$
f_{\bullet, *}: E_{*}^{p, q}\left(X_{\bullet}\right) \rightarrow E_{*}^{p, q}\left(Y_{\bullet}\right)
$$

On the $E_{1}$ pages, this gives commutative diagrams

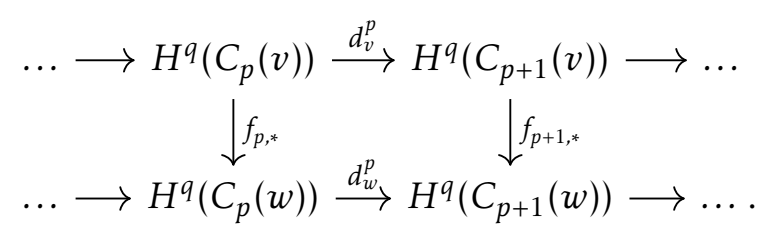

By Corollary 6.5, we get

$$
\operatorname{rk} E_{2}^{p, q}\left(f_{\bullet, *}\right)=\operatorname{rk}\left(\begin{array}{cc}
d_{v}^{p} & 0 \\
f_{p, *}-d_{w}^{p-1}
\end{array}\right)-\operatorname{rk}\left(d_{v}^{p}\right)-\operatorname{rk}\left(d_{w}^{p-1}\right) .
$$

By assumption and by Remark 4.2, the right hand side is independent of the Weil cohomology theory $H$, hence so is the left hand side. Then the same holds for the rank of $f_{\bullet, *}: H^{i}\left(C_{\bullet}(v)\right) \rightarrow H^{i}\left(C_{\bullet}(w)\right)$ since $f_{\bullet, *}$ respects the canonical splitting of (6.7), which proves (2) by diagram (6.5).

Remark 6.7. Instead of the diagrammatic argument given above, one would be tempted to use the strong version of the alterations result [dJo96].

This gives a proper hypercover $X_{\bullet} \rightarrow X$ along with an embedding $X_{\bullet} \rightarrow \bar{X}_{\bullet}$ such that each $\bar{X}_{n}$ is smooth projective, and the complement of $X_{n} \subseteq \bar{X}_{n}$ is a simple normal crossings divisor $D_{n}$.

Assuming $\operatorname{Rk}(Y, Z)$ holds for smooth projective $k$-schemes $Y$ and $Z$, a simplicial argument for $D_{n}$ shows that the dimension of $H_{\mathrm{c}}^{i}\left(X_{n}\right)$ (hence also $H^{i}\left(X_{n}\right)$ ) is independent of the Weil cohomology theory [Kat94, p. 29]. This again uses the Weil conjectures to conclude degeneration of a spectral sequence.

Then the spectral sequence for the hypercovering $X_{\bullet} \rightarrow X$ computes $H^{i}(X)$ in terms of $H^{i}\left(X_{n}\right)$. However, now the purity argument no longer applies, and we have no idea on what page the spectral sequence might 
degenerate. So even knowing $\operatorname{Rk}(Y, Z)$ for smooth quasi-projective $k$-schemes $Y$ and $Z$ does not imply $\operatorname{Dim}_{\mathrm{c}}(X)$ (or its variant $\operatorname{Dim}(X)$ for cohomology $H^{i}$ ) through this method.

The above argument is a way around this problem. The author is not aware of a place in the literature where this argument is carried out, but variants of it might have been known to experts.

Remark 6.8. To prove Theorem 6.6 (1) and (2) only in the case where $X$ and $Y$ are proper, one can carry out the proof above without talking about (simplicial) mapping cones. Indeed, applying the proof above to the morphism $f_{\bullet}: Y_{\bullet} \rightarrow X_{\bullet}$ (of simplicial schemes) instead of the morphism $f_{\bullet}: C_{\bullet}(v) \rightarrow C_{\bullet}(w)$ (of cosimplicial all-or-nothing motives) shows that the dimensions of $H^{i}(X)$ and $H^{i}(Y)$ as well as the rank of $f^{*}: H^{i}(X) \rightarrow H^{i}(Y)$ do not depend on $H$.

Applying this to the closed immersion $v: V \rightarrow \bar{X}$ as in (6.3), we see that

$$
\begin{aligned}
\operatorname{dim} H_{\mathrm{c}}^{i}(X) & =\operatorname{dim}\left(\operatorname{ker} H^{i}(v)\right)+\operatorname{dim}\left(\operatorname{coker} H^{i-1}(v)\right) \\
& =\operatorname{dim} H^{i}(\bar{X})-\operatorname{rk}\left(H^{i}(v)\right)+\operatorname{dim} H^{i-1}(V)-\operatorname{rk}\left(H^{i-1}(v)\right),
\end{aligned}
$$

so finally $\operatorname{dim} H_{\mathrm{c}}^{i}(X)$ does not depend on $H$. This recovers Theorem 6.6 (1) without ever using simplicial mapping cones. (The mapping cone of complexes is still implicitly used through Corollary 6.5.)

One can also obtain Theorem 6.6 (2) in this way (at least for étale or crystalline cohomology), by looking at the different weight parts as in (6.7) and applying Corollary 6.5 in the case where the complexes themselves are mapping cones.

This requires a different kind of axiom comparable to (A6), using the mapping cone of complexes instead. However, mapping cones in the derived category are only unique up to non-unique isomorphism, and to get a commutative diagram like (6.5) one needs to choose mapping cones functorially. We use the ones from simplicial schemes, but there are other possibilities. For example, Cisinki-Déglise's mixed Weil cohomologies [CD12] work in the dg setting, which may well be enough to make this part of the argument work.

\section{Dimensions and cycle class maps}

In this section, the ground field $k$ is allowed to be arbitrary again. We start with a Bertini irreducibility theorem. We do some extra work in Lemma 7.2 to avoid extending the base field, using the Bertini irreducibility theorem of Charles-Poonen [CP16] as well as the classical one [Jou83, Theorem 6.3(4)].

The main application of these Bertini theorems is Corollary 7.5, which we use to prove the implication $(3 \mathrm{~b}) \Rightarrow(1)$ of Theorem 4.6. The idea is that if $Z \subseteq X$ is an effective codimension $m$ cycle on a smooth projective variety $(X, H)$, then for $m$ general sections $H_{1}, \ldots, H_{m} \in|H|$ containing $Z$, the intersection $H_{1} \cap \ldots \cap H_{m}$ contains only one new component $Z^{\prime}$, which is smooth away from $Z$ (in particular reduced). Therefore,

$$
[Z]=H^{m}-\left[Z^{\prime}\right] \in \mathrm{CH}^{m}(X),
$$

which realises $[Z]$ as a difference of two irreducible cycles. We do something similar for an arbitrary (not necessarily effective) cycle $\alpha \in \mathrm{CH}^{m}(X)$.

We suggest the reader skip ahead to Corollary 7.5 on a first reading.

Definition 7.1. Let $X$ be a separated $k$-scheme of finite type. Consider the following condition on a closed subscheme $Z \subseteq X$ :

(Irr): For every irreducible component $X_{i}$ of $X_{\bar{k}}$, there exists a unique irreducible component $Z_{i}$ of $Z_{\bar{k}}$ contained in $X_{i}$, and moreover $Z_{i}=X_{i} \cap Z_{\bar{k}}$.

If $X$ is geometrically normal, then $X_{i} \cap X_{j}=\varnothing$ for $i \neq j$. In this case, the final statement of (Irr) is automatic: we clearly have $Z_{i} \subseteq X_{i} \cap Z_{\bar{k}}$, and all other components $Z_{j}$ of $Z_{\bar{k}}$ are disjoint from $X_{i} \cap Z_{\bar{k}}$. 
Lemma 7.2. Let $(X, H)$ be a projective $k$-scheme all of whose components have dimension $n \geq 2$, let $Z \subseteq X$ be a geometrically reduced closed subscheme of pure dimension $\ell \neq 0, n$, and let $Y_{1}, \ldots, Y_{s} \subseteq X$ be integral subschemes of dimension $<n$ that are not contained in $Z$. Assume that $X$ is smooth away from $Z$ and at the generic points of $Z$. Then for $d \gg 0$, there exists an element $D \in|d H|$ containing $Z$ such that

- $D$ is smooth away from $Z$;

- $D$ is smooth at the generic points of $Z$;

- the divisor $D \backslash Z$ of $X \backslash Z$ satisfies (Irr);

- $D$ does not contain any of the $Y_{j}$.

Proof. Write $X^{\text {sing }}$ and $Z^{\text {sing }}$ for the singular (non-smooth) loci of $X \rightarrow$ Spec $k$ and $Z \rightarrow$ Spec $k$ respectively. By assumption, $X \backslash X^{\text {sing }}$ contains $U=X \backslash Z$, as well as the generic points of $Z$. Since $Z$ is geometrically reduced, $Z^{\text {sing }}$ has dense open complement. Thus, the open subset $W=X \backslash\left(X^{\text {sing }} \cup Z^{\text {sing }}\right)$ is smooth of dimension $n$, contains $U$, and $W \cap Z$ is smooth and dense. Let $Y$ be the union of the zero-dimensional $Y_{j}$, and note that $Y \cap Z=\varnothing$.

If $k$ is infinite, choose $d \gg 0$ such that $\mathcal{I}_{Z}(d H)$ is globally generated, and let $V$ be the image of $H^{0}\left(X, \mathcal{I}_{Z}(d H)\right)$ in $H^{0}\left(X, \mathcal{O}_{X}(d H)\right)$, i.e. $|V|$ is the linear system in $|d H|$ of sections vanishing on $Z$. Since $\mathcal{I}_{Z}(d H)$ is globally generated, the base locus of $|V|$ is exactly $Z$, so $|V|$ defines a morphism

$$
\phi: U \rightarrow \mathbf{P}\left(V^{*}\right) \text {. }
$$

Note that $\phi$ is a composition of a locally closed immersion and a coordinate projection, hence unramified. Thus [Jou83, Corollary I.6.11(2,3)] shows that the locus of $D \in|V|=\mathbf{P}(V)(k)$ such that $D \cap U$ is smooth and satisfies (Irr) is dense open.

Since the $Y_{j}$ are not contained in $Z$, the locus of $D \in|V|$ not containing $Y_{j}$ is dense open. Similarly, the locus of $s \in H^{0}\left(X, \mathcal{I}_{Z}(d H)\right)$ whose restriction to $\mathcal{I}_{Z} / \mathcal{I}_{Z}^{2}(d H)$ is nonzero is dense open. Thus we can find a $k$-point of $\mathbf{P}(V)$ satisfying all desired properties, since a dense open subset of $\mathbf{P}(V)$ has a $k$-point if $k$ is infinite.

If $k$ is finite, we apply [Wut17, Theorem 2.1], where Wutz's $X, Y, Z, k, \ell, m$ are our $W, Y, Z, \ell-1, \ell, n$ respectively. This shows that the set

$$
\left\{\begin{array}{l|l}
D \in|d H| & \begin{array}{l}
Z \subseteq D \text { and } Y \cap D=\varnothing \\
\operatorname{dim}(D \cap W)_{\text {sing }} \leq \ell-1, \\
D \cap(X \backslash Z) \text { is smooth of dimension } n-1 .
\end{array}
\end{array}\right\}
$$

has positive density $\mu>0$ as $d \rightarrow \infty$. In particular, such $D$ are smooth at the generic points of $Z$, since $Z$ has (pure) dimension $\ell$. Applying Bertini's irreducibility theorem [CP16, Theorem 1.2] to $X \backslash Z$ shows that the set of $D$ such that $D \backslash Z$ satisfies (Irr) in $X \backslash Z$ has density 1 .

Finally, if $Y_{j}$ is positive-dimensional, then the ideal sheaf $\mathcal{I}_{Z \cap Y_{j}} \subseteq \mathcal{O}_{Y_{j}}$ is nonzero because $Y_{j} \nsubseteq Z$. The map $\mathcal{I}_{Z} \rightarrow \mathcal{I}_{Z \cap Y_{j}}$ is surjective by the second and third isomorphisms theorems. Hence, for $d \gg 0$, the map

$$
\phi_{d}: H^{0}\left(X, \mathcal{I}_{Z}(d)\right) \rightarrow H^{0}\left(Y_{j}, \mathcal{I}_{Z \cap Y_{j}}(d)\right)
$$

is surjective. The dimension of the right hand side is (eventually) a polynomial of degree $\operatorname{dim} Y_{j}>0$ in $d$, so

$$
\text { codim } \operatorname{ker}\left(\phi_{d}\right) \rightarrow \infty \quad \text { as } d \rightarrow \infty \text {. }
$$

Hence, the functions that vanish on $Y_{j}$ have density 0 as $d \rightarrow \infty$. Therefore, the intersection of the three sets has positive density $\mu$.

Lemma 7.3. Let $k$ be a perfect field, and let $(X, H)$ be a smooth projective $k$-scheme all of whose components have dimension $n$. Let $Z_{1}, \ldots, Z_{r} \subseteq X$ and $Y_{1}, \ldots, Y_{s} \subseteq X$ be pairwise distinct integral subschemes of codimension $m \neq 0, n$. Then for $d_{1}, \ldots, d_{m} \gg 0$, there exist sections $D_{1}, \ldots, D_{m}$ of $\left|d_{1} H\right|, \ldots,\left|d_{m} H\right|$ intersecting properly such that 


$$
\left[\bigcap_{i=1}^{m} D_{i}\right]=\sum_{i=1}^{r}\left[Z_{i}\right]+[Z],
$$

where $Z \subseteq X$ is (geometrically) reduced, satisfies (Irr), does not contain any of the $Z_{i}$ and $Y_{j}$, and $Z \backslash \cup Z_{i}$ is smooth.

Proof. Let $Z^{\prime}=\bigcup Z_{i}$. We apply Lemma 7.2 inductively on the codimension $m$ of the $Z_{i}$ to find sections $D_{i}$ of $\left|d_{i} H\right|$ containing $Z^{\prime}$ such that $\bigcap D_{i}$ is smooth away from $Z^{\prime}$ and at the generic points of $Z^{\prime}$ and does not contain any of the $Y_{j}$, and $\cap D_{i} \backslash Z^{\prime}$ satisfies (Irr). Thus the multiplicity of $\cap D_{i}$ at the generic points of $Z^{\prime}$ is 1 , so

$$
\left[\bigcap_{i=1}^{m} D_{i}\right]=\sum_{i=1}^{r}\left[Z_{i}\right]+\alpha
$$

for an effective cycle $\alpha$ none of whose components is contained in $Z^{\prime}$.

If $Z$ is the closure of $\bigcap D_{i} \backslash Z^{\prime}$, then restricting to $X \backslash Z^{\prime}$, we find that $\alpha=[Z]$, where all coefficients are 1 since $\cap D_{i} \backslash Z^{\prime}$ is smooth. Then $Z$ is geometrically reduced by [Stacks, Tag 020I]. Finally, $Z$ satisfies (Irr) because $\cap D_{i} \backslash Z^{\prime}$ does, and it does not contain any of the $Y_{j}$ since $\cap D_{i} \backslash Z^{\prime}$ does not contain any $Y_{j} \backslash Z^{\prime}$ by the choice of the $D_{i}$.

Theorem 7.4. Let $k$ be a perfect field, let $(X, H)$ be a smooth projective $k$-scheme of equidimension $n$, let $\alpha \in \mathrm{CH}^{m}(X)$ be a pure dimensional cycle, and let $e_{0} \in \mathbf{Z}$. If $m \neq 0, n$, then there exists a (geometrically) reduced subscheme $Z \subseteq X$ satisfying (Irr) and $e \geq e_{0}$ such that

$$
\alpha=[Z]-e H^{m} \in \mathrm{CH}^{m}(X) .
$$

Proof. Write $\alpha=\sum_{i} n_{i}\left[Z_{i}\right]-\sum_{i} n_{i}^{\prime}\left[Z_{i}^{\prime}\right]-e H^{m}$, where $Z_{i}, Z_{i}^{\prime} \subseteq X$ are pairwise distinct integral subschemes of codimension $m$, and $n_{i}, n_{i}^{\prime} \in \mathbf{Z}_{\geq 0}$ (to start with, we may take $e=0$ ). We will apply Lemma 7.3 a few times.

First, by induction on $z=\sum\left(n_{i}-1\right)+\sum\left(n_{i}^{\prime}-1\right)$, we will reduce to the case where $z=0$. Indeed, if $z>0$, then one of the $n_{i}$ or $n_{i}^{\prime}$ is $\geq 2$. Say $n_{1} \geq 2$; the case $n_{i}^{\prime} \geq 2$ is similar. Applying Lemma 7.3 to $Z_{1}$ with $\left\{Y_{j}\right\}:=\left\{Z_{i} \mid i>1\right\} \cup\left\{Z_{i}^{\prime}\right\}$, we can write $\left[Z_{1}\right]=d H^{m}-\left[Z^{\prime}\right]$, where $Z^{\prime}$ does not contain any of the $Z_{i}$ and $Z_{i}^{\prime}$, and $Z^{\prime}$ is generically smooth (in particular reduced). Adjoining the irreducible components of $Z^{\prime}$ to the $Z_{i}^{\prime}$ and changing $e$ to $e-d$, we have reduced $z$ by one, because the new components coming from $Z^{\prime}$ all have coefficient 1 . After finitely many steps, we get $z=0$, so all $n_{i}$ and $n_{i}^{\prime}$ are equal to 1 .

Now applying Lemma 7.3 to the $Z_{i}$ while avoiding the $Z_{i}^{\prime}$, we get a generically smooth subscheme $Z^{\prime} \subseteq X$ of codimension $m$ and such that $\sum_{i}\left[Z_{i}\right]=d H^{m}-\left[Z^{\prime}\right]$. Adjoining the components of $Z^{\prime}$ to the $Z_{i}^{\prime}$ and replacing $e$ by $e-d$, we can write

$$
\alpha=-\sum_{i}\left[Z_{i}^{\prime}\right]-e H^{m}
$$

Finally, applying Lemma 7.3 to the $Z_{i}^{\prime}$, for every $d_{1}, \ldots, d_{m} \gg 0$ we get a geometrically reduced subscheme $Z$ of codimension $m$ satisfying (Irr) such that $d H^{m}=\sum_{i}\left[Z_{i}^{\prime}\right]+[Z]$, where $d=\prod d_{i}$. Thus,

$$
\alpha=[Z]-(e+d) H^{m} \text {. }
$$

Choosing the $d_{i}$ large enough so that $e+d \geq e_{0}$ gives the result.

Corollary 7.5. Let $k$ be a perfect field, let $(X, H)$ be a smooth projective $k$-scheme of equidimension $n$, and let $\alpha \in \mathrm{CH}^{m}(X)$. If $m \neq 0$, $n$, then there exist geometrically reduced subschemes $Z_{1}, Z_{2} \subseteq X$ satisfying (Irr) such that

$$
\alpha=\left[Z_{1}\right]-\left[Z_{2}\right] \in \mathrm{CH}^{m}(X) .
$$

Proof. By Theorem 7.4, we may write $\alpha=\left[Z_{1}\right]-e H^{m}$, where $Z_{1}$ satisfies (Irr) and $e$ may be taken arbitrarily large. Applying the usual Bertini irreducibility theorem [Jou83, Theorem 6.10(4)], [CP16, Corollary 1.4] inductively, we find a subscheme $Z_{2} \subseteq X$ satisfying (Irr) with $\left[Z_{2}\right]=e H^{m}$. Indeed, over an infinite field we 
can do this for any $e$ [Jou83, Theorem 6.10(4)], whereas over a finite field the positivity of the density [CP16, Corollary 1.4] shows that there exists $e_{0}$ such that for all $e \geq e_{0}$ we can find a member satisfying (Irr).

Theorem 7.6. Let $k$ be an algebraically closed field. Let X be a smooth projective $k$-scheme, and assume that $\operatorname{Dim}_{\mathrm{c}}(U)$ holds for every open subscheme $U \subseteq X$. Then $\mathrm{Cl}(X)$ holds.

Proof. Since $\mathrm{CH}_{\mathbf{Q}}^{*}(U \amalg V)=\mathrm{CH}_{\mathbf{Q}}^{*}(U) \times \mathrm{CH}_{\mathbf{Q}}^{*}(V)$, and the same statement holds for the cohomology ring, we may assume $X$ is irreducible of dimension $n$, hence (geometrically) integral. Let $\alpha \in \mathrm{CH}_{\mathbf{Q}}^{*}(X)$ be given. Because the cycle class map is homogeneous, it suffices to treat the case where $\alpha \in \mathrm{CH}_{\mathbf{Q}}^{m}(X)$ is of pure dimension $d=n-m$. If $m=0$ or $m=n$, then clearly the kernels of the cycle class maps to $H^{m}(X)$ and $\mathcal{H}^{m}(X)$ agree.

Now assume $m \neq 0, n$. Then by Corollary 7.5, we may write $\alpha=\left[Z_{1}\right]-\left[Z_{2}\right]$, where $Z_{1}$ and $Z_{2}$ are reduced subschemes satisfying (Irr); since $X$ is integral this just means that $Z_{1}$ and $Z_{2}$ are integral as well. If $\alpha=0$ there is nothing to prove, so we may assume $\left[Z_{1}\right] \neq\left[Z_{2}\right]$. Let $Z=Z_{1} \cup Z_{2} \subseteq X$, let $U=X \backslash Z$, and consider the long exact cohomology sequence with compact support of (A1):

$$
\ldots \rightarrow H_{\mathrm{c}}^{i}(U) \rightarrow H_{\mathrm{c}}^{i}(X) \rightarrow H_{\mathrm{c}}^{i}(Z) \rightarrow \ldots
$$

By (A2), we have $\operatorname{dim} H_{\mathrm{c}}^{2 d}(Z)=2$ and $H_{\mathrm{c}}^{i}(Z)=0$ for $i>2 d$. Thus, we have an exact sequence

$$
\ldots \rightarrow H_{\mathrm{c}}^{2 d}(X) \stackrel{i^{*}}{\rightarrow} H_{\mathrm{c}}^{2 d}(Z) \rightarrow H_{\mathrm{c}}^{2 d+1}(U) \rightarrow H_{\mathrm{c}}^{2 d+1}(X) \rightarrow 0 .
$$

By (A2) again, the map $i^{*}: H_{\mathrm{c}}^{2 d}(X) \rightarrow H_{\mathrm{c}}^{2 d}(Z) \cong K \oplus K$ is the dual of

$$
\begin{aligned}
K \oplus K & \longrightarrow H^{2 m}(X) \\
(\lambda, \mu) & \longmapsto \lambda \operatorname{cl}\left(Z_{1}\right)+\mu \mathrm{cl}\left(Z_{2}\right) .
\end{aligned}
$$

If $h$ is an ample divisor class on $X$, then $h^{d} \cdot Z_{i}>0$. Therefore, $\operatorname{cl}\left(Z_{i}\right) \neq 0$, so the rank of $i^{*}$ is either 1 or 2 . Additivity of dimensions in (7.1) gives

$$
\operatorname{rk}\left(i^{*}\right)=\operatorname{dim} H_{\mathrm{c}}^{2 d}(Z)-\operatorname{dim} H_{\mathrm{c}}^{2 d+1}(U)+\operatorname{dim} H_{\mathrm{c}}^{2 d+1}(X) .
$$

By assumption, $\operatorname{dim} H_{\mathrm{c}}^{2 d+1}(U)$ and $\operatorname{dim} H_{\mathrm{c}}^{2 d+1}(X)$ are independent of the Weil cohomology theory $H$, hence so is the rank of $i^{*}$.

We now claim that $\mathrm{cl}_{H}(\alpha)=0$ if and only if $\operatorname{rk}\left(i^{*}\right)=1$ and $h^{d} \cdot Z_{1}=h^{d} \cdot Z_{2}$. Indeed, if $\operatorname{cl}(\alpha)=0$, then $\left(i^{*}\right)^{\vee}$ has a kernel, so $i^{*}$ cannot have rank 2. Moreover, cupping the relation $\operatorname{cl}\left(Z_{1}\right)=\operatorname{cl}\left(Z_{2}\right)$ with $h^{d}$ gives $h^{d} \cdot Z_{1}=h^{d} \cdot Z_{2}$. Conversely, if $\operatorname{rk}\left(i^{*}\right)=1$ and $h^{d} \cdot Z_{1}=h^{d} \cdot Z_{2}$, then there is a unique $\left[\lambda_{H}: \mu_{H}\right] \in \mathbf{P}^{1}(K)$ such that

$$
\lambda_{H} \mathrm{cl}_{H}\left(Z_{1}\right)=\mu_{H} \mathrm{cl}_{H}\left(Z_{2}\right)
$$

Again, cupping with $h^{d}$ gives $\lambda_{H} Z_{1} \cdot h^{d}=\mu_{H} Z_{2} \cdot h^{d}$, forcing $\left[\lambda_{H}: \mu_{H}\right]=[1: 1]$, so that $\operatorname{cl}_{H}(\alpha)=0$.

Because the rank of $i^{*}$ and the intersection numbers $h^{d} \cdot Z_{i}$ are independent of the Weil cohomology theory $H$, this shows that the vanishing of $\mathrm{cl}_{H}(\alpha)$ is also independent of $H$.

Corollary 7.7. Let $k$ be an algebraically closed field. If $\operatorname{Dim}_{c}(X)$ holds for every smooth quasi-projective $k$-scheme $X$, then $\mathrm{Cl}(X)$ holds for every smooth projective $k$-scheme $X$.

\section{References}

[Ber74] P. Berthelot, Cohomologie cristalline des schémas de caractéristique $p>0$, Lecture Notes in Mathematics, vol. 407, Springer-Verlag, Berlin-New York, 1974.

[Ber86] P. Berthelot, Géométrie rigide et cohomologie des variétés algébriques de caractéristique p. In: Introductions aux cohomologies $p$-adiques (Luminy, 1984), Mém. Soc. Math. France 23 (1986), $7-32$. 
[Ber97] P. Berthelot, Dualité de Poincaré et formule de Künneth en cohomologie rigide, C. R. Acad. Sci. Paris Sér. I Math. 325 (1997), no. 5, 493-498.

[BS15] B. Bhatt and P. Scholze, The pro-étale topology for schemes, Astérisque 369 (2015), 99-201.

[CP16] F. Charles and B. Poonen, Bertini irreducibility theorems over finite fields, J. Amer. Math. Soc. 29 (2016), no. 1, 81-94.

[CD12] D.-C. Cisinski and F. Déglise, Mixed Weil cohomologies, Adv. Math. 230 (2012), no. 1, 55-130.

[CD19] D.-C. Cisinski and F. Déglise, Triangulated categories of mixed motives, Springer Monographs in Mathematics, Springer, Cham, 2019.

[Del74a] P. Deligne, La conjecture de Weil. I. Inst. Hautes Études Sci. Publ. Math. 43 (1974), 273-307.

[Del74b] P. Deligne, Théorie de Hodge. III. Inst. Hautes Études Sci. Publ. Math. 44 (1974), 5-77.

[Del80] P. Deligne, La conjecture de Weil. II. Inst. Hautes Études Sci. Publ. Math. 52 (1980), 137-252.

[GM87] H. Gillet and W. Messing, Cycle classes and Riemann-Roch for crystalline cohomology, Duke Math. J. 55 (1987), no. 3, 501-538.

[Gro85] M. Gros, Classes de Chern et classes de cycles en cohomologie de Hodge-Witt logarithmique, Mém. Soc. Math. France 21 (1985), 1-87.

[Gro69] A. Grothendieck, Standard conjectures on algebraic cycles. In: Algebraic Geometry (Internat. Colloq., Tata Inst. Fund. Res., Bombay, 1968), pp. 193-199, Oxford Univ. Press, London, 1969.

[Ill94] L. Illusie, Crystalline cohomology. In: Motives (Seattle, WA, 1991), pp. 43-70, Proc. Sympos. Pure Math., vol. 55, part 1, Amer. Math. Soc., Providence, RI, 1994.

[Ill06] L. Illusie, Miscellany on traces in -adic cohomology: a survey, Jpn. J. Math. 1 (2006), no. 1, 107-136.

[Jan92] U. Jannsen, Motives, numerical equivalence, and semi-simplicity, Invent. Math. 107 (1992), no. 3, $447-452$.

[dJo96] A. J. de Jong, Smoothness, semi-stability and alterations, Inst. Hautes Études Sci. Publ. Math. 83 (1996), 51-93.

[Jou83] J.-P. Jouanolou, Théorèmes de Bertini et applications, Progress in Mathematics, vol. 42, Birkhäuser, Boston, MA, 1983.

[Kat94] N. M. Katz, Review of $\ell$-adic cohomology. In: Motives (Seattle, WA, 1991), pp. 21-30, Proc. Sympos. Pure Math., vol. 55, part 1, Amer. Math. Soc., Providence, RI, 1994.

[KM74] N. M. Katz and W. Messing, Some consequences of the Riemann hypothesis for varieties over finite fields, Invent. Math. 23 (1974), 73-77.

[Kle68] S. L. Kleiman, Algebraic cycles and the Weil conjectures. In: Dix exposés sur la cohomologie des schémas, pp. 359-386, Adv. Stud. Pure Math., vol. 3, North-Holland, Amsterdam, 1968.

[Kle72] S. L. Kleiman, Motives. In: Algebraic geometry, Oslo 1970 (Proc. Fifth Nordic Summer School in Math.), pp. 53-82, Wolters-Noordhoff, Groningen, 1972.

[Kle94] S. L. Kleiman, The standard conjectures. In: Motives (Seattle, WA, 1991), pp. 3-20, Proc. Sympos. Pure Math., vol. 55, part 1, Amer. Math. Soc., Providence, RI, 1994.

[LeS11] B. Le Stum, The overconvergent site, Mém. Soc. Math. France 127 (2011).

[Nag62] M. Nagata, Imbedding of an abstract variety in a complete variety, J. Math. Kyoto Univ. 2 (1962), 1-10. 
[Nag63] M. Nagata, A generalization of the imbedding problem of an abstract variety in a complete variety, J. Math. Kyoto Univ. 3 (1963), 89-102.

[Sam60] P. Samuel, Relations d'équivalence en géométrie algébrique. In: Proc. Internat. Congress Math. 1958, pp. 470-487, Cambridge Univ. Press, New York, 1960.

[Sch94] A. J. Scholl, Classical motives. In: Motives (Seattle, WA, 1991), pp. 163-187, Proc. Sympos. Pure Math., vol. 55, part 1, Amer. Math. Soc., Providence, RI, 1994.

[SGA4 $\left.{ }_{\mathrm{I}}\right] \quad$ Séminaire de Géométrie Algébrique du Bois-Marie 1963-64. Théorie des topos et cohomologie étale des schémas (SGA 4). Tome 1: Théorie des topos. Dirigé par M. Artin, A. Grothendieck, et J. L. Verdier. Lecture Notes in Mathematics, vol. 269, Springer-Verlag, Berlin-New York, 1972.

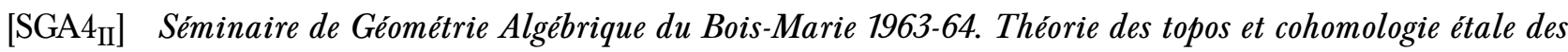
schémas (SGA 4). Tome 2. Dirigé par M. Artin, A. Grothendieck et J. L. Verdier. Lecture Notes in Mathematics, vol. 270, Springer-Verlag, Berlin-New York, 1972.

[SGA4 III] Séminaire de Géométrie Algébrique du Bois-Marie 1963-64. Théorie des topos et cohomologie étale des schémas (SGA 4). Tome 3. Dirigé par M. Artin, A. Grothendieck et J. L. Verdier. Lecture Notes in Mathematics, vol. 305, Springer-Verlag, Berlin-New York, 1973.

[SGA41/2] P. Deligne, Séminaire de géométrie algébrique du Bois-Marie. Cohomologie étale (SGA 41/2). Lecture Notes in Mathematics, vol. 569, Springer-Verlag, Berlin, 1977.

[Stacks] The Stacks project Authors. The Stacks project, 2005-2020.

[Tsu03] N. Tsuzuki, Cohomological descent of rigid cohomology for proper coverings, Invent. Math. 151 (2003), no. 1, 101-133.

[Wut17] F. Wutz, The singular locus of hypersurface sections containing a closed subscheme over finite fields, preprint, 2017. arXiv:1704.08108 